\title{
Eisenhart lifts and symmetries of time-dependent systems
}

\author{
M. Cariglia ${ }^{1,2 *}$ C. Duval ${ }^{3 \dagger}$, G. W. Gibbons ${ }^{4,5 \ddagger}$, P. A. Horvathy ${ }^{5,6 \S}$ \\ ${ }^{1}$ DEFIS, Universidade Federal de Ouro Preto, MG-Brasil \\ ${ }^{2}$ Dipartimento di Fisica, \\ Universitá degli Studi di Camerino, Italy \\ ${ }^{3}$ Centre de Physique Théorique, \\ Aix Marseille Université \& Université de Toulon \& CNRS UMR 7332, \\ Case 907, 13288 Marseille, France \\ ${ }^{4}$ D.A.M.T.P., Cambridge University, U.K. \\ ${ }^{5}$ Laboratoire de Mathématiques et de Physique Théorique, \\ Université de Tours (France) \\ 6 Institute of Modern Physics, \\ Chinese Academy of Sciences, \\ Lanzhou, China
}

(Dated: May 20, 2016)

\footnotetext{
* e-mail:marco@iceb.ufop.br

† Aix-Marseille Université, CNRS, CPT, UMR 7332, 13288 Marseille, France. mailto:duval@cpt.univmrs.fr

$\ddagger$ mailto:G.W.Gibbons@damtp.cam.ac.uk

$\S$ mailto:horvathy@lmpt.univ-tours.fr
} 


\begin{abstract}
Certain dissipative systems, such as Caldirola and Kannai's damped simple harmonic oscillator, may be modelled by time-dependent Lagrangian and hence time dependent Hamiltonian systems with $n$ degrees of freedom. In this paper we treat these systems, their projective and conformal symmetries as well as their quantisation from the point of view of the Eisenhart lift to a Bargmann spacetime in $n+2$ dimensions, equipped with its covariantly constant null Killing vector field. Reparametrization of the time variable corresponds to conformal rescalings of the Bargmann metric. We show how the Arnold map lifts to Bargmann spacetime. We contrast the greater generality of the Caldirola-Kannai approach with that of Arnold and Bateman. At the level of quantum mechanics, we are able to show how the relevant Schrödinger equation emerges naturally using the techniques of quantum field theory in curved spacetimes, since a covariantly constant null Killing vector field gives rise to well defined one particle Hilbert space.

Time-dependent Lagrangians arise naturally also in cosmology and give rise to the phenomenon of Hubble friction. We provide an account of this for Friedmann-Lemaitre and Bianchi cosmologies and how it fits in with our previous discussion in the non-relativistic limit.
\end{abstract}

Keywords: Caldirola-Kanai model, damped oscillator, Bargmann space, Eisenhart lift, Hubble model, Dmitriev-Zel'dovich equations 


\section{Contents}

$\begin{array}{ll}\text { I. Introduction } & 4\end{array}$

II. Generalized Caldirola-Kanai systems 6

A. Relation to Rayleigh's dissipation function 7

B. The Eisenhart lift 8

$\begin{array}{ll}\text { C. Projective point of view } & 10\end{array}$

D. Generalized Caldirola-Kanai models on group manifolds 11

E. Schrödinger equation via the wave equation $\quad 15$

F. Pseudo-stationary states 16

G. Second quantised QFT approach 17

III. Symmetries of damped oscillators $\quad 19$

A. The Arnold transformation $\quad 19$

1. Symmetries of the CK oscillator 21

B. The Bateman "Doppelgänger" (Bateman double) 23

1. Symmetries of the Bateman system 24

IV. Hubble Friction 26

A. Particle moving in an inhomogeneous cosmological spacetime 27

B. The Dmitriev-Zel'dovich equations 28

C. Bianchi cosmology 29

$\begin{array}{ll}\text { Acknowledgments } & 30\end{array}$

$\begin{array}{ll}\text { References } & 30\end{array}$ 


\section{INTRODUCTION}

Over the past few years the extended spacetime originally proposed by Eisenhart [1], then forgotten, and rediscovered [2-5] under the name of "Bargmann structure", has provided a powerful framework from which to view and explore the symmetries of various naturally occurring Lagrangian systems, both classical and quantum. See e.g. [6] for a recent review.

Hitherto, the majority of such studies have been cases for which the Lagrangian $L$ is independent of time $t$. As a consequence, the equations of motion are an autonomous set of ordinary differential equations of what can be thought of as an isolated system. Energy is conserved and the time evolution map $f_{t}$ (assuming that it is defined for all $t$ ) is classically by means of a one-parameter group of canonical transformations, and quantum-mechanically by means of a one-parameter group of unitary transformations. In both cases, the time evolution map satisfies the group composition law

$$
f_{t+t^{\prime}}=f_{t^{\prime}} \circ f_{t}
$$

By contrast, if the Lagrangian $L$ is time dependent the equations of motion are nolonger autonomous, they contain the time explicitly. The system is no longer isolated and may be thought of as coupled to an external environment. Energy is no longer conserved, and since, to the extent that there is no preferred time parameter, the motion may be regarded by means of a path in the space of canonical transformations, and quantummechanically by means of a path in the space of unitary transformations. This is a reflection of the fact that since there is no preferred choice of time parameter for such systems, one is alway free to replace $t$ by a monotonic function $\tau(t)$ of $t$. For the time independent case there is, up to an affine transformation, a unique parameter $t$ for which (I.1) is true.

If what-would-be-the-energy in the absence of time dependence decreases monotonically for the time dependent case, it is natural to regard the system as dissipative due to friction which transfers energy to some other, possibly microscopic degrees of freedom. However in general what would be the energy in the absence of time dependence may both increase or decrease. This might happen if the system were subject to external time-dependent forces or torques designed by an external agent to move the system in a different way from its natural motion for which $L$ is time-independent. This sort of situation arises in the subject of control theory both at the classical and the quantum level. A recent application is given in [7].

The original motivation of the present study arose from an enquiry to one of us from the authors of [8-11] how their work on the symmetries of the damped simple harmonic oscillator fits into the Eisenhart framework. Although on the face of it a comparatively simple problem, the literature on the damped simple harmonic oscillator is very extensive, no doubt because of its wide range of applications in all areas of science and technology. It would be quite out of place to review all of it here. The reader is advised to consult, e.g., $[12,13]$ and references therein. 
Roughly speaking, one finds that there are two main approaches to the problem to which we refer as the Caldirola-Kannai $[14,15]$ and the Bateman approach [16].

In the former one constructs a time dependent Lagrangian whose equations of motion are those of the damped oscillator:

$$
\ddot{x}+\gamma \dot{x}+\omega^{2} x=0,
$$

and then to pass to a time-dependent Hamiltonian

$$
H=\frac{1}{2 m} e^{-\gamma t} p^{2}+\frac{1}{2} e^{\gamma t} m \omega^{2} x^{2} .
$$

One may then pass to the quantum theory in the standard fashion by replacing $p$ by $\frac{1}{i} \frac{\partial}{\partial x}$ in (I.3) The resulting Hamiltonian operator $\hat{H}$ is manifestly time dependent and does not commute with the time derivative,

$$
\left[\hat{H}, \frac{\partial}{\partial t}\right] \neq 0 \text {. }
$$

This has caused some comments in the literature, but it appears to presents no fundamental difficulty to developing the theory. Moreover there is no difficulty in extending the procedure to a wide variety of time dependent Lagrangians representing a particle moving on a curved configuration space in the presence a potential function and such that both the metric giving the kinetic terms and the potential term may depend on both time and space and the Euler-Lagrange equations are non-linear in positions, unlike (I.2). As we shall show in $\S \mathrm{II}$, there is no difficulty in constructing the Eisenhart lift.

We are then able to show that the problem of quantizing the system may then be achieved by regarding it as one of constructing a free quantum field theory in a curved Lorentzian spacetime background given by the Eisenhart lift.

It is well known that for a general curved space time there is no privileged notion of positive frequency and hence no well defined notion of a time independent one particle Hilbert space upon which to build the full Hilbert space by the the usual Fock construction. However the Eisenhart lift is not a general a Lorentzian spacetime, but rather it admits a Bargmann structure $[2,5]$ and in particular a null Killing vector field. It has been known for some time [17] that in this situation there is a privileged notion of positive frequency and hence a privileged one-particle Hilbert space. It is this Hilbert space which corresponds to the Caldirola-Kannai construction in this general case.

In the case of a linear equation of motion, such as that of the damped simple harmonic oscillator such as (I.2) there is a procedure due to Arnold [18] of mapping the problem to that of a free particle by means of a mapping of the associated non-relativistic space time $t, x$ into itself. The authors of [8] have shown how this classical Arnold map may be made the basis of a quantum mechanical Arnold map to map the quantum algebra of the classical free particle into the quantum algebra of the damped simple harmonic oscillator. We are able to show that the Arnold maps, both classical and quantum, may be lifted to the Eisenhart/Bargmann space where, since in this special case, it is conformally flat, they act as conformal transformations. 
The second approach is that of Bateman [16] in which one introduces an additional degree of freedom $y$ to the simple harmonic oscillator (I.2) which we call the Bateman double (or "Doppelgänger") and a time dependent Lagrangian form which follows the original equation of motion (I.2) and that of its Doppelgänger

$$
\ddot{y}-\gamma \dot{y}+\omega^{2} y=0 .
$$

Note that the Doppelgänger is anti-damped rather than damped. However, as a consequence of the time-independence of the the Lagrangian, there is a total conserved energy but this energy is not positive definite. Physically the Doppelgänger represents the environment into which the original oscillator loses energy such that what the original particle loses the environment gains. The authors of [9] have related the Caldirola-Kannai and Bateman approaches and how the algebra of their asymmetries map into one another using the Arnold maps. Using the Eisenhart lift we are able to lift these maps and view the symmetries and their relation in terms of conformal geometry

From our work it appears that both the Arnold and the Bateman procedures have a limited range of applications since in their straightforward form they can be used only when the equations of motion are linear. By contrast we have seen that the CaldirolaKannai approach has a much wider range of applicability.

Having described these basic examples we turn to further applications and extensions of the Eisenhart lifting procedure. A standard dynamical model, productive of many symmetries, is that of free or geodesic motion on a Lie group with respect to a leftinvariant metric. We briefly review the necessary formalism and then discuss its extension to the case when both the metric, and any added potential may depend upon time. An interesting example is provided by the case of a planar isotropic harmonic oscillator without friction. In this case the Eisenhart lift is to a bi-invariant metric on the fourdimensional Cangemi-Jackiw group [19], a spacetime also known in string theory as the Nappi-Witten [20] solution.

Our final applications are to the phenomenon of Hubble friction. In the case it is the expanding universe which constitutes the source of time dependence in the Lagrangian for particles moving in a background Friedmann-Lemaitre-Robertson-Walker universe. Going beyond isotropy, in the final section we incorporate anisotropy by considering the spatial metric to be a time-dependent but left-invariant metric on one of Bianchi's nine three-dimensional Lie groups.

\section{GENERALIZED CALDIROLA-KANAI SYSTEMS}

Consider the time-dependent Lagrangian and Hamiltonian

$$
\left\{\begin{array}{l}
L=\frac{m}{2 \alpha(t)} g_{i j}\left(x^{k}\right) \dot{x}^{i} \dot{x}^{j}-\beta(t) V\left(x^{i}, t\right), \\
H=\frac{\alpha(t)}{2 m} g^{i j}\left(x^{k}\right) p_{i} p_{j}+\beta(t) V\left(x^{i}, t\right),
\end{array}\right.
$$


respectively, where $g_{i j}\left(x^{k}\right) d x^{i} d x^{j}$ is a positive metric on a curved configuration space $Q$ with local coordinates $x^{i}$, where $i=1, \ldots, n$. We denote by $m$ the mass of the system. The coefficients $\alpha(t)$ and $\beta(t)$ depend on time $t$ and $V\left(x^{i}, t\right)$ is some (possibly time-dependent) scalar potential. The associated Lagrange equations yield the equations of motion

$$
\frac{d^{2} x^{i}}{d t^{2}}+\Gamma_{j k}^{i} \frac{d x^{j}}{d t} \frac{d x^{k}}{d t}-\frac{\dot{\alpha}}{\alpha} \frac{d x^{i}}{d t}=-\frac{\alpha \beta}{m} g^{i j} \partial_{j} V,
$$

where the $\Gamma_{j k}^{i}$ are the Christoffel symbols of the Levi-Civita connection of the metric $g_{i j}$. When the system is explicitly time-dependent, the energy is not conserved.

Setting, for example,

$$
n=1, \quad V=\frac{1}{2} m \omega^{2} x^{2}, \quad \alpha=\beta^{-1}=e^{-\gamma t},
$$

we obtain the damped harmonic oscillator [8-16],

$$
L=\frac{m}{2} e^{\gamma t}\left(\left|\frac{d \boldsymbol{x}}{d t}\right|^{2}-\omega^{2} \boldsymbol{x}^{2}\right), \quad \frac{d^{2} \boldsymbol{x}}{d t^{2}}+\gamma \frac{d \boldsymbol{x}}{d t}=-\omega^{2} \boldsymbol{x} .
$$

Returning to the general case (II.7), we note that the "frictional [or anti-frictional] term" $-(\dot{\alpha} / \alpha) \dot{x}^{i}$ can be eliminated by introducing a new time-parameter $\tau=\varphi(t)$ defined by

$$
d \tau=\alpha d t
$$

which carries (II.7) into the form

$$
\frac{d^{2} x^{i}}{d \tau^{2}}+\Gamma_{j k}^{i} \frac{d x^{j}}{d \tau} \frac{d x^{k}}{d \tau}=-\frac{\beta}{m \alpha} g^{i j} \partial_{j} V .
$$

A similar argument applies also in the more general case when $\Gamma_{j k}^{i}$ in (II.7) is any linear connection on $Q$. Thus, whether there is friction, depends crucially on which timescale or system of clocks that we use. It is worth mentioning that time reparametrization, (II.10), can also be viewed in terms of projective connections, see Section II C.

\section{A. Relation to Rayleigh's dissipation function}

It is traditional [21], when considering linear, dissipative systems with constant coefficients to introduce what is known as Rayleigh's dissipation function ${ }^{1}$. Consider, for example, the system of electric circuits governed by the equations

$$
L_{i j} \frac{d^{2} q^{j}}{d t^{2}}+R_{i j} \frac{d q^{j}}{d t}+P_{i j} q^{j}=0
$$

with $P_{i j}=C_{i j}^{-1}$, where, for electric circuits, $\dot{q}^{j}=i^{j}$ is the electric current flowing through the $j$ 'th circuit. $L_{i j}, R_{i j}$ and $C_{i j}$ are the components of the mutual inductance, resistance

${ }^{1}$ Minguzzi [22] has recently considered non-linear versions. 
and capacitance matrices, respectively, which are assumed symmetric and independent of time and $q^{j}$. The matrix whose components are $P_{i j}$ is sometimes known as the elastance matrix. One may be more ambitious and consider electrical machinery in which case one has, in addition to electrical components such as inductors, resistors and capacitors, various rigid bodies with generalized coordinates $q^{i}$, with mass matrices and spring constants etc. The combined system may also be described using equations (II.12), where some of the components $L_{i j}$ are masses and $P_{i j}$ spring constants. For simplicity we shall assume that $\operatorname{det} L_{i j} \neq 0$ and that there are no generalized forces on the r.h.s of (II.12).

The standard treatment is then to define the kinetic energy $T=\frac{1}{2} L_{i j} \dot{q}^{i} \dot{q}^{j}$, the potential energy $V=\frac{1}{2} P_{i j} q^{i} q^{j}$ and Rayleigh's dissipation function $R=\frac{1}{2} R_{i j} \dot{q}^{i} \dot{q}^{j}$ and write the equations of motion as

$$
\frac{d}{d t}\left(\frac{\partial T}{\partial \dot{q}^{i}}\right)+\frac{\partial V}{\partial q^{i}}=-\frac{\partial R}{\partial \dot{q}^{i}}=-R_{i j} \dot{q}^{j}
$$

Thus

$$
\frac{d}{d t}(T+V)=2 R
$$

Introducing $L=T-V^{2}$ leads to the equation:

$$
\frac{d}{d t}\left(\frac{\partial L}{\partial \dot{q}^{i}}\right)-\frac{\partial L}{\partial q^{i}}=-\frac{\partial R}{\partial \dot{q}^{i}} .
$$

This is not of Lagrangian form and it is not clear how to construct a Hamiltonian. The electrical engineer Kron attempted to interpret what is going on as some sort of nonRiemannian geometry [23]. However so far this view point does not seem to have been widely adopted. Our strategy is to adjoin two additional coordinates to the generalized coordinates $q^{j}$ and give an interpretation in terms of pseudo-Riemannian geometry. If (as it typically happens), the inductance matrix $L_{i j}$ has only strictly positive eigenvalues, it will be Lorentzian geometry. It is possible that Kron's non-Riemannian geometry may have a place in that context.

Following Caldirola and Kanai we consider the equation ${ }^{3}$

$$
\frac{d}{d t}\left(A_{i j} \dot{q}^{j}\right)+B_{i j} q^{j}=0
$$

with $A=F^{-1} L$ for a time-dependent matrix $F$. We find $-\dot{F} F^{-1} L=R, B=F^{-1} P$, and so $F$ satisfies

$$
\dot{F}+R L^{-1} F=0 .
$$

If there is only one degree of freedom we recover the result of Caldirola and Kanai [14, 15].

\section{B. The Eisenhart lift}

Further insight can be gained by lifting the problem to one higher dimension called "Bargmann space" [2, 5]. In order to Eisenhart-lift the system (II.6), we introduce a

\footnotetext{
${ }^{2}$ In this paragraph $L$ should be distinguished from the inductance matrix $L$ whose components are $L_{i j}$.

${ }^{3}$ from now on $L$ reverts to being the inductance matrix $L$ whose components are $L_{i j}$.
} 
spacetime extension parametrized by $\left(x^{a}\right)=\left(x^{i}, t, s\right)$, endowed with a Brinkmann metric $[5,24]$ of the form

$$
\mathrm{g}_{a b} d x^{a} d x^{b}=\frac{1}{\alpha} g_{i j} d x^{i} d x^{j}+2 d t d s-\frac{2 \beta V}{m} d t^{2} .
$$

Denoting by $\left(p_{a}\right)=\left(p_{i}, p_{t}, p_{s}\right)$ the conjugate momentum and putting $p_{t}=-H$ and $p_{s}=m$, we easily find

$$
\frac{1}{2 m} \mathrm{~g}^{a b} p_{a} p_{b}=\frac{\alpha}{2 m} g^{i j} p_{i} p_{j}-H+\beta V=0,
$$

using the contravariant form of the metric. This confirms the null character of $\left(p_{a}\right)$ and matches the form (II.6) of the Hamiltonian : the null-geodesics of the metric (II.18) project to spacetime as the solutions of the original equations of motion (II.7).

Our clue is now that the time reparametrization $\varphi: t \rightarrow \tau$ defined by (II.10) which allowed us to eliminate the friction amounts to a conformal rescaling of the metric,

$$
\mathrm{g}_{a b}=\frac{1}{\alpha} \tilde{\mathrm{g}}_{a b}, \quad \tilde{\mathrm{g}}_{a b} d \tilde{x}^{a} d \tilde{x}^{b}=g_{i j}\left(x^{k}\right) d x^{i} d x^{j}+2 d \tau d s-\frac{2 \beta}{m \alpha} V d \tau^{2},
$$

where $\left(\tilde{x}^{a}\right)=\left(x^{i}, \tau, s\right)$, and $\alpha, \beta$ are now viewed as functions of $\tau$. Setting e.g.,

$$
n=2, \quad g_{i j}=\delta_{i j} \quad \text { and } \quad V=\frac{1}{2} m \omega^{2}\left(x^{2}+y^{2}\right)
$$

in (II.19), then the metric $\tilde{\mathrm{g}}_{a b} d \tilde{x}^{a} d \tilde{x}^{b}$ may be identified as a special case of the metric (7.1) in [25]. One sets $\zeta=x+i y, v=2 s, u=\tau, f(u, \zeta)=0$ and $\phi(u, \zeta)=\sqrt{\frac{\beta}{\alpha}} \zeta$. For example, the metric and two-form

$$
\begin{aligned}
\tilde{\mathrm{g}}_{a b} d \tilde{x}^{a} d \tilde{x}^{b} & =d \zeta d \bar{\zeta}+d \tau(2 d s+H(\tau, \zeta, \bar{\zeta}) d \tau), \\
F & =d \tau \wedge\left(\partial_{\zeta} \phi d \zeta+\partial_{\bar{\zeta}} \bar{\phi} d \bar{\zeta}\right)
\end{aligned}
$$

where

$$
H(\tau, \zeta, \bar{\zeta})=f(\tau, \zeta)+\bar{f}(\tau, \bar{\zeta})-2 \phi(\tau, \zeta) \bar{\phi}(\tau, \bar{\zeta})
$$

is a solution of the Einstein-Maxwell equations if $f$ and $\phi$ are arbitrary functions of $\tau$ and holomorphic in $\zeta$, see [25].

If $n=2, g_{i j}=\delta_{i j}, f=0$ and $\phi=B(\tau) \zeta$ here, then eqn (II.22) and eqn (II.19) agree when

$$
V=\frac{m \alpha}{\beta} B^{2}(\tau) \zeta \bar{\zeta}
$$

On the other hand, if $B(\tau)$ is a constant, then $\tilde{\mathrm{g}}_{a b} d \tilde{x}^{a} d \tilde{x}^{b}$ is the left-invariant metric on the Cangemi-Jackiw group [19, 20], see [25] and sec. II D for further discussion. If

$$
B^{2}(\tau)=\frac{\beta}{\alpha} \frac{\omega^{2}}{2}
$$

with $\left.\beta=\alpha^{-1}=(1-\gamma \tau)\right)^{-1}$, then we recover the Caldirola-Kanai oscillator.

Since (II.21)-(II.22) is a solution of the Einstein-Maxwell equations with vanishing cosmological constant, the Ricci scalar of the metric $\tilde{g}_{a b} d \tilde{x}^{a} d \tilde{x}^{b}$ vanishes. Since $\widetilde{\nabla}^{a} \alpha \widetilde{\nabla}_{a} \alpha=$ 
0 and $\widetilde{\nabla}^{a} \widetilde{\nabla}_{a} \alpha$ both vanish, the Ricci scalar of the conformally related metric $g_{a b}$ in (II.19) also vanishes. This has the consequence that the massless scalar wave equation we shall discuss in Secs. D, E and $\mathrm{F}$ is unambiguous, since there can be no $R \phi$ term [26].

For the choice $n=2, g_{i j}=\delta_{i j}, f=0$ and $\phi=B(\tau) \zeta$ made above, the Weyl tensor of (II.21) vanishes since $\partial_{\zeta}^{2} H=0$ [25] and is therefore conformally flat, consistently with [27]. All conformally flat Bargmann spaces with $n=2$ were determined in [28].

We conclude this subsection by explaining the relation to another recently proposed approach [29]. The $(2 n+3)$-dimensional "evolution space" [30] of a spinless particle of mass $m$ moving in our $(n+2)$-dimensional Bargmann manifold $(M, g, \xi)$ is $N \subset T^{*} M$, defined by the constraint

$$
p_{a} \xi^{a}=m \text {. }
$$

cf. [5]. Putting $\xi=\partial_{s}$ we have $p_{s}=m$; the induced canonical Cartan 1-form on $N$ is therefore

$$
\alpha=p_{i} d x^{i}-H d t+m d s .
$$

The motions which in fact null geodesics of the Bargmann metric are the leaves of the characteristic distribution of the two-form induced by $d \alpha$ on the submanifold $p_{a} p^{a}=0$ of $N$. $\alpha$ is a contact 1 -form on the evolution space. Putting $S=m s$ as a genuine action coordinate, we readily end-up with Eq. \# (68) of [29]. Their contact structure is therefore the canonical one on $N$, our Bargmann evolution space.

\section{Projective point of view}

Earlier, in sec. II we have seen that the time-dependent friction term on the 1.h.s. of eqn (II.7) may be eliminated by means of the change of the time parameter eqn (II.10) to produce (II.11). In this equation the $\Gamma_{j k}^{i}$ are the components of the Levi-Civita connection of the time-independent metric $g_{i j}$ in (II.6). However, we may consider a more general equation in which $\Gamma_{j k}^{i}$ are the components of a general linear affine connection. One may even even further generalize the situation to the case when $\Gamma^{i}{ }_{j k}$ depend on time. If we consider $\tau$ as an extra coordinate so that $x^{\mu}=\left(\tau, x^{i}\right)$ then eqn (II.11) is a special case of a general equation of an affinely parametrized auto-parallel curve of the form

$$
\frac{d^{2} x^{\rho}}{d \lambda^{2}}+\Gamma_{\mu \nu}^{\rho} \frac{d x^{\mu}}{d \lambda} \frac{d x^{\nu}}{d \lambda}=0
$$

To obtain (II.11) we set

$$
\Gamma_{j k}^{i}=\text { Christoffel symbols of } g_{i j}, \quad \Gamma_{\mu \nu}^{0}=0, \quad \Gamma_{00}^{i}=\frac{\beta}{m \alpha} g^{i j} \partial_{j} V .
$$

Solutions of (II.27) are called geodesics or auto-parallel curves of the affinely connected manifold $M^{1+n}$ whose local coordinates are $x^{\mu}$. A priori $M^{1+n}$ need not be equipped with a metric (Lorentzian or otherwise).

The manifold $M^{1+n}$ is said to be projectively flat if its geodesics may be mapped into straight lines in $\mathbb{R}^{1+n}$. If $n>1$, a necessary and sufficient condition for this is 
that the Weyl projective curvature tensor of the affine connection $\Gamma_{\mu \nu}^{\rho}$, denoted by $W_{\beta \gamma \delta}^{\alpha}$ vanishes, see Thm 3.1 of [31]. For a NC connection whose only nonzero components are $\Gamma_{00}^{i}=-g^{i}(\boldsymbol{x}, t)$ with $i=1, \ldots, n$, there holds $W_{\beta \gamma \delta}^{\alpha}=0$ iff

$$
\mathbf{g}(\boldsymbol{x}, t)=a(t) \boldsymbol{x}+\mathbf{b}(t)
$$

implying a $\operatorname{SL}(n+2, \mathbb{R})$-symmetry for the projective geodesics. It follows that for an isotropic simple harmonic oscillator the Weyl projective tensor vanishes. The group $\mathrm{SL}(n+2, \mathbb{R})$ does not preserve the conformal Galilei structure in general, though, and requiring it to do so, we end up with the Schrödinger group; see [10, 32] for details.

The case $n=1$ is special in that $W_{\beta \gamma \delta}^{\alpha}$ vanishes identically. However, a special case of Thm 3.4 of [31] states that the equation

$$
\ddot{x}+c(x, t) \dot{x}+g(x, t)=0
$$

may be brought to the form

$$
\widetilde{x}^{\prime \prime}=0
$$

by a suitable transformation $\widetilde{x}=\widetilde{x}(x, t)$ and $\widetilde{t}=\widetilde{t}(x, t)$ (where the prime means $d / d \widetilde{t}$ ), provided

$$
3 \frac{\partial^{2} g}{\partial x^{2}}-2 \frac{\partial^{2} c}{\partial t \partial x}-2 c \frac{\partial c}{\partial x}=0 \quad \text { and } \quad \frac{\partial^{2} c}{\partial x^{2}}=0 .
$$

This equation is automatically satisfied for a damped harmonic oscillator with possibly time- (but not space) dependent friction term and frequency. This explains the $\operatorname{SL}(3, \mathbb{R})$ symmetry discovered in [33].

The general solution of eqn. (II.32) is give by

$$
c(x, t)=A(t) x+B(t), \quad g(x, t)=\frac{A^{2}}{9} x^{3}+\frac{1}{3}(\dot{A}+A B) x^{2}+C(t) x+D(t) .
$$

We remark en passant that Liénard's non-linear oscillator with position-dependent damping [34],

$$
\ddot{x}+f^{\prime}(x) \dot{x}+x=0,
$$

which includes van der Pol's oscillator [35] as a special case, $f=x^{3}-x$, admits projective symmetry i.e., may be brought to the form of a free particle only if $f^{\prime}(x)=\gamma$ with $\gamma=$ const..

\section{Generalized Caldirola-Kanai models on group manifolds}

A wide variety of interesting dynamical models may be obtained by taking the configuration space $Q$ to be a Lie group $G$ with local coordinates $q^{i}$. That is, there are group elements $G \ni g=g\left(q^{i}\right)$, and left and right invariant Cartan-Maurer forms

$$
g^{-1} d g=\lambda^{a} \mathbf{e}_{a}, \quad d g g^{-1}=\rho^{a} \mathbf{e}_{a}
$$


such that if $\mathbf{e}_{a}$ is a basis for the Lie algebra $\mathfrak{g}$ satisfying

$$
\left[\mathbf{e}_{a}, \mathbf{e}_{b}\right]=C_{a}{ }^{c}{ }_{b} \mathbf{e}_{c},
$$

then

$$
d \lambda^{c}=-\frac{1}{2} C_{a}{ }^{c}{ }_{b} \lambda^{a} \wedge \lambda^{b}, \quad d \rho^{c}=\frac{1}{2} C_{a}{ }^{c}{ }_{b} \rho^{a} \wedge \rho^{b} .
$$

We denote the canonical Darboux coordinates on the co-tangent space $T^{\star} G=G \times \mathfrak{g}^{\star}$ by $\left(q^{i}, p_{i}\right)$.

The left and right invariant vector field $L_{a}^{i}$ and $R_{a}^{i}$ dual to $\lambda_{i}^{a}, \rho_{i}^{a}$ respectively,

$$
\lambda_{i}^{a} L_{b}^{i}=\delta_{b}^{a}, \quad \rho_{i}^{a} R_{b}^{i}=\delta_{b}^{a},
$$

satisfy

$$
\left[L_{a}, L_{b}\right]=C_{a}{ }^{c}{ }_{b} L_{c}, \quad\left[R_{a}, L_{b}\right]=0, \quad\left[R_{a}, R_{b}\right]=-C_{a}{ }^{c}{ }_{b} R_{c}
$$

and generate right and left translations on $G$, respectively. One may define moment maps into the dual $\mathfrak{g}^{\star}$ of the Lie algebra by

$$
M_{a}=p_{i} L_{a}^{i}, \quad N_{a}=p_{i} R_{a}^{i},
$$

with Poisson brackets

$$
\left\{M_{a}, M_{b}\right\}=-C_{a}{ }^{b}{ }_{c} M_{b}, \quad\left\{M_{a}, N_{b}\right\}=0, \quad\left\{N_{a}, N_{b}\right\}=C_{a}{ }^{b}{ }_{c} M_{b},
$$

which generate the lifts of right and left translation to $T^{\star} G$. A Hamiltonian $H=H\left(q^{i}, p_{i}\right)$ which is left-invariant therefore satisfies

$$
\dot{N}_{a}=\left\{N_{a}, H\right\}=0,
$$

and so the moment maps $N_{a}$ are constants of the motion. By contrast, the moment maps generating right actions, $M_{a}$, are in general time-dependent,

$$
\dot{M}_{a}=\left\{M_{a}, H\right\} \neq 0 \text {. }
$$

A left-invariant Lagrangian may be constructed from combinations of left-invariant velocities or angular velocities,

$$
\omega^{a}=\lambda_{i}^{a} \dot{q}^{i},
$$

giving a Hamiltonian which is a combination of the moment maps $M_{a}$,

$$
H=H\left(M_{a}\right) .
$$

Thus (II.43) provide an autonomous 1st order system of ODE's on $\mathfrak{g}^{\star}$ for the moment maps $M_{a}$ called the Euler equations. To obtain the motion on the group, one uses the equation $\dot{q}^{i}=\partial H / \partial p_{i}$. Now

$$
p_{i}=M_{a} \lambda_{i}^{a} \quad \text { and so } \quad \dot{q}^{i}=L_{a}^{i} \frac{\partial H}{\partial M_{a}} .
$$


The method described above can reasonably be called hodographic. Hamilton [36] defined the hodograph of a particle motion $\boldsymbol{x}=\boldsymbol{x}(t)$ in $\mathbb{E}^{3}$ as the the curve described by the vector $\mathbf{v}(t)=d \boldsymbol{x} / d t$, a construction very similar to the Gauss map for surfaces in $\mathbb{E}^{3}$. Hamilton then discovered [36] the elegant result that the hodograph for Keplerian motion is a circle.

Since velocity space and momentum space are naturally identified in this case we may think about the motion in phase space $T^{\star} \mathbb{E}^{3}=(\boldsymbol{x}, \mathbf{p})$, and then observe that because $\mathbb{E}^{3}$ is flat, there is, in addition to the standard vertical projection $(\boldsymbol{x}, \mathbf{p}) \rightarrow(\boldsymbol{x}, 0)$, a well defined horizontal map or hodographic projection $(\boldsymbol{x}, \mathbf{p}) \rightarrow(0, \mathbf{p})$. For a general configuration space $Q$, the co-tangent manifold $T^{\star} Q$ will not admit a well-defined horizontal projection. However if $Q=G$, a group manifold, then it does, and the Euler equations govern the motion of the hodograph.

The simplest models of this type correspond to geodesic motion with respect to a left-invariant metric on $G$ of the form

$$
\mathrm{g}_{i j} d q^{i} d q^{j}=B_{a b} \lambda^{a} \lambda^{b}
$$

where $B_{a b}$ is a symmetric bi-linear form on $\mathfrak{g}$. In this case

$$
T=\frac{1}{2} B_{a b} \omega^{a} \omega^{b}, \quad H=\frac{1}{2} B^{a b} M_{a} M_{b}
$$

Usually the metric considered is positive definite, but it need not be. For examples of spacetimes which may be thought of as group manifolds see [37] and also below.

So far we have been considering metrics for which the bilinear form $B_{a b}$ is time dependent but from the previous section it is clear that we may consider it to be timedependent, $B_{a b}=B_{a b}(t)$. Everything will go through as before except that our Hamilton's and Euler's equations will no longer be autonomous. The case of $G=S O(3)$ is familiar as giving the free motion of a rigid body with one point fixed. The bi-linear form $B_{a b}$ is then the inertia quadric. Our generalization would apply if it were time dependent.

A further generalization would be to let the fixed point move, in which case we would replace $S O(3)$ by the Euclidean group $E(3)$. The Bianchi group $V I I_{0}$ has been applied to the optical geometry of the ground state of chiral nematics [38]. The time-dependent theory might well have relevance in that case as well.

As an illustration, we discuss briefly the symmetries of the Cangemi-Jackiw-NappiWitten metric, eqn (II.50) below. The Nappi-Witten (or diamond) group has originally been proposed as space-time for lineal gravity [19] and turned out to be an exact string vacuum [20]. It is the semidirect product of $\mathrm{SO}(2)$ which represents compactified time with $H(1)$, the Heisenberg group in 1 dimension,

$$
G=\widetilde{\mathrm{SE}}(2)=\mathrm{SO}(2) \ltimes H(1) .
$$

The clue is that $G$ carries a natural $(3+1)$-dimensional curved Bargmann structure [39]

$$
g=d x \otimes d x+d y \otimes d y+d t \otimes d s+d s \otimes d t-\omega^{2}\left(x^{2}+y^{2}\right) d t \otimes d t, \quad \xi=\frac{\partial}{\partial s},
$$


where the generator, $\xi$, of the centre, $\mathrm{SO}(2)$, of $H(1)$ is null and covariantly constant for the Lorentz metric $g$; here $\omega \neq 0$ is a free parameter. Thus (II.50) yields a $(2+1)$ dimensional Galilei structure on the "base manifold", which is in fact the Euclidean group, $\mathrm{SE}(2)=\mathrm{SO}(2) \ltimes \mathbb{R}^{2}$, obtained by factoring out the centre of $H(1)$.

The Lie algebra of infinitesimal isometries of $(G, g)$ can be found by integrating the Killing equations, yielding the following 7 generators

$$
\begin{aligned}
J & =x \partial_{y}-y \partial_{x}, \\
X_{1} & =\cos (\omega t) \partial_{x}+\omega x \sin (\omega t) \partial_{s}, \\
X_{2} & =\sin (\omega t) \partial_{x}-\omega x \cos (\omega t) \partial_{s}, \\
Y_{1} & =\cos (\omega t) \partial_{y}+\omega y \sin (\omega t) \partial_{s}, \\
Y_{2} & =\sin (\omega t) \partial_{y}-\omega y \cos (\omega t) \partial_{s}, \\
T & =\partial_{t}, \\
S & =\partial_{s} .
\end{aligned}
$$

The nontrivial Lie brackets are

$$
\begin{aligned}
& {\left[J, X_{i}\right]=-Y_{i}, \quad\left[J, Y_{i}\right]=X_{i}, \quad\left[X_{1}, X_{2}\right]=\left[Y_{1}, Y_{2}\right]=-\omega S,} \\
& {\left[X_{i}, T\right]=\omega \epsilon_{i j} X_{j},\left[Y_{i}, T\right]=\omega \epsilon_{i j} Y_{j},}
\end{aligned}
$$

where $i, j=1,2$. Notice that $S$ being central, the isometries form the "Bargmann group" of the NW group viewed as a Bargmann manifold, and thus automatically project onto "spacetime" SE(2) as the 6-dimensional "Galilei" group. This group of ultimately projects in turn onto "time", $\mathrm{SO}(2)$, as translations.

Viewing things differently, let us recall the group law for the NW-group $G$ with topology $S^{1} \times \mathbb{R}^{2} \times S^{1}$ :

$$
(\phi, \mathbf{X}, z) \cdot\left(\phi^{\prime}, \mathbf{X}^{\prime}, z^{\prime}\right)=\left(\phi+\phi^{\prime}, R(\phi) \mathbf{X}^{\prime}+\mathbf{X}, z+z^{\prime}-\frac{1}{2} \mathbf{X} \cdot J R(\phi) \mathbf{X}^{\prime}\right)
$$

where $(J \mathbf{X})_{i}=\epsilon_{i j} X_{j}$, and $R(\phi)=\exp (\phi J) \in \mathrm{SO}(2)$. The left-invariant Maurer-Cartan 1-form $\Theta=(\tau, \boldsymbol{\theta}, \varpi)$ then reads

$$
\tau=d \phi, \quad \boldsymbol{\theta}=R(-\phi) d \mathbf{X}, \quad \varpi=\frac{1}{2} \mathbf{X} \cdot J d \mathbf{X}+d z .
$$

One can furthermore check that

$$
\tilde{g}=\theta^{1} \otimes \theta^{1}+\theta^{2} \otimes \theta^{2}+\varpi \otimes \tau+\tau \otimes \varpi, \quad \tilde{\xi}=\frac{\partial}{\partial z},
$$

endows the NW-group $G$ with a canonical Bargmann structure.

Left translations preserve (by definition) the Maurer-Cartan form, $L_{a}^{*} \Theta=\Theta$, and also the "vertical" vector, $L_{a}^{*} \tilde{\xi}=\tilde{\xi}$, whereas right translations only preserve the Lorentz metric, $R_{a}^{*} \tilde{g}=\tilde{g}$, and the "vertical" vector, $R_{a}^{*} \tilde{\xi}=\tilde{\xi}$, for all $a \in G$. The group $G \times G$ thus acts isometrically on itself according to

$$
\Phi\left(a^{\prime}, a^{\prime \prime}\right)(a)=a^{\prime} \cdot a \cdot\left(a^{\prime \prime}\right)^{-1}
$$


with $a, a^{\prime}, a^{\prime \prime} \in G$. But this action is not effective, $\operatorname{ker}(\Phi) \cong \mathbb{R}$, the centre of $G$ diagonally embedded in $G \times G$. Hence $\operatorname{Isom}(G, \tilde{g})=(G \times G) / \mathbb{R}$.

Explicitly, the structure (II.55) on $G$ parametrized by $(\phi, \mathbf{X}=(\tilde{x}, \tilde{y}), z)$ is

$$
\tilde{g}=d \tilde{x} \otimes d \tilde{x}+d \tilde{y} \otimes d \tilde{y}+\varpi \otimes d \phi+d \phi \otimes \varpi, \quad \tilde{\xi}=\frac{\partial}{\partial z}
$$

where $\varpi=\frac{1}{2}(\tilde{x} d \tilde{y}-\tilde{y} d \tilde{x})+d z$ is as in (II.54).

Let us mention that the Bargmann structure (II.57) of the NW-group (II.49) is related to the original one in (II.50) by the diffeomorphism $(t, x, y, s) \mapsto(\phi, \tilde{x}, \tilde{y}, z)$ of $G$ where

$$
\phi=2 \omega t, \quad \tilde{x}=x \cos (\omega t)+y \sin (\omega t), \quad \tilde{y}=-x \sin (\omega t)+y \cos (\omega t), \quad z=\frac{s}{2 \omega} .
$$

The infinitesimal generators of the left action of $G$ :

$$
\begin{aligned}
J^{\prime} & =\partial_{\phi}+\tilde{y} \partial_{\tilde{x}}-\tilde{x} \partial_{\tilde{y}}, \\
X_{1}^{\prime} & =\partial_{\tilde{x}}-\frac{1}{2} \tilde{y} \partial_{z}, \\
X_{2}^{\prime} & =\partial_{\tilde{y}}+\frac{1}{2} \tilde{x} \partial_{z}, \\
Z^{\prime} & =\partial_{z},
\end{aligned}
$$

commute with those of the right action of $G$ :

$$
\begin{aligned}
J^{\prime \prime} & =\partial_{\phi}, \\
X_{1}^{\prime \prime} & =\cos \phi \partial_{\tilde{x}}-\sin \phi \partial_{\tilde{y}}+\frac{1}{2}(\tilde{y} \cos \phi+\tilde{x} \sin \phi) \partial_{z}, \\
X_{2}^{\prime \prime} & =\sin \phi \partial_{\tilde{x}}+\cos \phi \partial_{\tilde{y}}+\frac{1}{2}(\tilde{y} \sin \phi-\tilde{x} \cos \phi) \partial_{z}, \\
Z^{\prime \prime} & =\partial_{z},
\end{aligned}
$$

yielding the 7 infinitesimal isometries $J^{\prime}, J^{\prime \prime}, X_{1}^{\prime}, X_{1}^{\prime \prime}, X_{2}^{\prime}, X_{2}^{\prime \prime}, Z^{\prime}=Z^{\prime \prime}$ corresponding, via (II.58), to those given in (II.51).

These results are consistent with those in [25], obtained in a different coordinate system.

The Nappi-Witten model has been generalized by Yang-Baxter deformations [40] however the sigma-model metric, i.e., the pp-wave (II.50), remains unchanged.

Finally, it is worth mentioning that an extension of this framework to infinite dimensional groups allows one to discuss hydrodynamics along similar terms [41].

\section{E. Schrödinger equation via the wave equation}

An issue which often crops up in the literature [12] has been what is the relevant Schrödinger equation for a time-dependent or frictional system. This is easily resolved using the Eisenhart lift. We take the massless minimally coupled scalar wave equation in our extended spacetime

$$
\frac{1}{\sqrt{-g}} \partial_{a}\left(\sqrt{-g} g^{a b} \partial_{b} \phi\right)=0
$$


where the metric, $g_{a b}$, is as in (II.18). Now, in our case $-g=-\operatorname{det} g_{a b}=\alpha^{-n} \operatorname{det} g_{i j}$, and we set $\phi=e^{i m s} \chi\left(x^{j}, t\right)$ as in [2] to find that the above wave equation reads

$$
i \partial_{t} \chi=-\frac{\alpha}{2 m} \nabla^{2} \chi+i \frac{n}{4} \frac{\dot{\alpha}}{\alpha} \chi+\beta V \chi
$$

where $\nabla^{2}$ is the Laplacian with respect to the metric $g_{i j}$ and $\dot{\alpha}=d \alpha / d t$. Agreement with equation (1) of [9] and equation (3) of [10], is obtained by putting $n=1 g_{11}=1$ and $\alpha=\beta^{-1}=e^{-\gamma t}$, with $\gamma$ a constant independent of time.

Substituting

$$
\chi=\alpha^{\frac{n}{4}} \Psi\left(x^{j}, t\right),
$$

in the general eqn (II.62), we may bring the system into the time-dependent Schrödinger form,

$$
i \partial_{t} \Psi=-\frac{\alpha}{2 m} \nabla^{2} \Psi+\beta V \Psi .
$$

An adaption of the standard calculation shows that

$$
\partial_{t}(\bar{\Psi} \Psi)=i \frac{\alpha}{2 m} \nabla^{j}\left(\bar{\Psi} \partial_{j} \Psi-\Psi \partial_{j} \bar{\Psi}\right)
$$

where $\nabla^{i}$ is the Levi-Civita covariant derivative of the metric $g_{i j}$. It follows that, modulo suitable boundary conditions, the conserved probability is

$$
\langle\Psi \mid \Psi\rangle=\int|\Psi|^{2} \sqrt{\operatorname{det} g_{i j}} d^{n} x,
$$

where the integral is taken for $t=$ const.. Moreover the quantum Hamiltonian

$$
\hat{H}=-\frac{\alpha}{2 m} \nabla^{2}+\beta V
$$

is self-adjoint with respect to the inner product $\langle\Psi \mid \Psi\rangle$. However, because of the timedependence of $\alpha$ and $\beta$ while the time dependence preserves the inner product $\langle\Psi \mid \Psi\rangle$, that is it consists of a one parameter family of unitarity maps, the one parameter family is not a one parameter subgroup of the unitary group. In other words,

$$
\Psi\left(t+t^{\prime}\right) \neq e^{-i \hat{H} t} \Psi\left(t^{\prime}\right)
$$

\section{F. Pseudo-stationary states}

According to [12] p. 24, for the under-damped simple harmonic oscillator in one spatial dimension the Schrödinger equation has a set of normalised solutions w.r.t. the inner product (II.66) called pseudo-stationary states of the form

$$
\Psi_{k}(x, t)=\frac{1}{\sqrt{2^{k} k !}}\left(\frac{m \Omega}{\pi}\right)^{\frac{1}{4}} e^{\gamma t} e^{-i\left(k+\frac{1}{2}\right) \Omega t} e^{-\frac{1}{2} m \Omega x^{2} e^{2 \gamma t}} H_{k}\left(\sqrt{m \Omega} x e^{\gamma t}\right),
$$

where $\Omega=\sqrt{\omega^{2}-\gamma^{2}}, k=0,1 \ldots$ and $H_{k}(y)$ is a Hermite polynomial. The general solution is therefore

$$
\Psi=\sum_{k} c_{k} \Psi_{k}(x, t)
$$


where the $c_{k}$ may be determined, for example, by expanding $\Psi(x, 0)$ as a series in the complete set of functions (II.69).

If $\gamma=0$, then the $\Psi_{k}$ are eigenstates of the Hamiltonian operator $\hat{H}$ and as a consequence (II.69) and (II.70) give the standard unitary evolution by means of a oneparameter subgroup of unitary transformations $U_{t}=e^{-i \hat{H} t}$. That is,

$$
\Psi(x, t)=U_{t} \Psi(x, 0)
$$

However when $\gamma \neq 0$, while (II.71) remains true, it is clear from (II.69) that the timeevolution given by (II.70) is not given by a one-parameter subgroup of unitary transformations, because $U_{t+t^{\prime}}=U_{t} \circ U_{t^{\prime}}$ is not satisfied.

\section{G. Second quantised QFT approach}

In the previous section we adopted a first quantised approach in which we simply took the classical minimally coupled wave equation and dimensionally reduced to obtain our candidate Schrödinger equation in the lower dimensional Newton-Cartan spacetime. One might ask how this is related to second quantised free Quantum Field Theory in the higher dimensional Bargmann spacetime. We would then face the basic problem of extending Poincaré invariant Quantum Field Theory to a fixed curved spacetime: the lack of a general acceptable unique definition of positive frequency and hence particle. Physically this ambiguity is responsible for the phenomenon of pair creation. Mathematically it means that in general there is no unique one particle Hilbert space $\mathcal{H}_{1}$ from which to give a Fock space construction of the entire sum of multi-particle Hilbert spaces $\mathcal{H}_{N}$

$$
\mathcal{H}=\sum_{N=0}^{\infty} \oplus \mathcal{H}_{N}
$$

where $\mathcal{H}_{0}$ is the no particle state. Following the precedent set in [17] we may tackle this problem making use of the null Killing vector field.

The inner product on $\mathcal{H}_{1}$ is usually defined using the conserved Klein-Gordon current

$$
J_{a}\left[\phi^{\prime}, \phi\right]=i\left(\bar{\phi}^{\prime} \partial_{a} \phi-\phi \partial_{a} \bar{\phi}^{\prime}\right)
$$

where $\phi$ and $\phi^{\prime}$ are two complex valued solutions of the massless minimally coupled scalar field eqn (II.61). There is no problem with the conservation, that is we have

$$
\nabla_{a} J^{a}=\frac{1}{\sqrt{-g}}\left(\partial_{a} \sqrt{-g} g^{a b} J_{b}\right)=0
$$

where $\nabla^{a}$ is the Levi-Civita covariant derivative with respect to the Bargmann metric $g_{a b}$. An inner product on the space of complex solutions may be defined by

$$
\left(\phi^{\prime}, \phi\right)=\int_{\Sigma} J^{a} d \Sigma_{a}
$$


where $\Sigma$ is a spacelike or possibly null Cauchy hypersurface. The problem is that the inner product so defined is indefinite unless the complex solutions are suitably restricted.

Suppose we have such a complete set of positive frequency functions $p_{i}$ such that

$$
\left(p_{i}, p_{j}\right)=\delta_{i j}, \quad\left(\bar{p}_{i}, p_{j}\right)=0, \quad\left(\bar{p}_{i}, \bar{p}_{j}\right)=-\delta_{i j},
$$

where $\{i\}$ is a suitable index set. Then the quantum field $\hat{\phi}$ may be expanded as

$$
\hat{\phi}=\sum_{i} \hat{a}_{i} p_{i}+\hat{a}_{i}^{\dagger} \bar{p}_{i}, \quad \text { where } \quad\left[\hat{a}_{i}, \hat{a}_{j}\right]=0, \quad\left[\hat{a}_{i}, \hat{a}_{j}^{\dagger}\right]=\delta_{i j}, \quad\left[\hat{a}_{i}^{\dagger}, \hat{a}_{j}^{\dagger}\right]=0 .
$$

The associated one particle Hilbert space $\mathcal{H}_{1}$ now consists of states

$$
|i\rangle=\hat{a}_{i}^{\dagger}|0\rangle
$$

where the associated no-particle state $|0\rangle$ satisfies

$$
\hat{a}_{i}|0\rangle=0 \text {. }
$$

In a general spacetime, there is no canonical choice of the basis $p_{i}$ and hence no canonical choice of no-particle state. That is because a function judged to be positive frequency at one time will not be judged to be positive frequency at a later time. One needs to introduce two bases $p_{i}^{\text {in }}$ and and $p_{i}^{\text {out }}$ say. In general one finds that

$$
\hat{a}_{i}^{\text {out }}=\alpha_{i j} \hat{a}_{j}^{\text {in }}+\beta_{i j} \hat{a}_{j}^{\text {in } \dagger}
$$

and thus the expected number of out particles in the in vacuum is

$$
N_{i}=\left\langle\text { in }\left|\hat{a}_{i}^{\text {out } \dagger} \hat{a}_{j}^{\text {out }}\right| \text { in }\right\rangle=\sum_{j}\left|\beta_{i j}\right|^{2} .
$$

If the spacetime admits a globally defined timelike or null Killing vector field $K^{a}$ one may restrict them to be linear combinations of positive (or negative) frequency solutions, that is for which

$$
K^{a} \partial_{a} \phi=-i \omega \phi
$$

and $\omega$ is positive (respectively negative). For a Bargmann metric we may always take

$$
K^{a} \partial_{a}=\frac{\partial}{\partial s}
$$

In [17] this choice was shown to imply that plane gravitational wave spacetimes, a particular example of a Bargmann type spacetime, do not give rise to pair-creation.

We may follow the same strategy in the present case. We choose as our Cauchy surfaces ${ }^{4}$, the null surfaces $t=$ constant. Since

$$
\partial_{a}\left(\sqrt{-g} J^{a}\right)=0
$$

\footnotetext{
${ }^{4}$ Strictly speaking they may not be Cauchy surfaces in the exact sense of the word, since null rays parallel to the $t=$ constant surfaces need not intersect them. In the present case that amounts to excluding solutions with zero frequency with respect to $s$.
} 
we need to integrate

$$
\int d s \int \sqrt{-g} J^{t} d^{n} x
$$

For example the case of the damped simple harmonic oscillator

$$
p_{k, m}=\frac{1}{\sqrt{2 \pi}} e^{-i m s} \Psi_{k}(x, t), \quad \sum_{i} \longleftrightarrow \sum_{k} \int_{s} d s
$$

where the $m$ is taken as the frequency [3]. We find

$$
\left(p_{k, m}, p_{k^{\prime}, m^{\prime}}\right)=\delta_{k k^{\prime}} \delta\left(m-m^{\prime}\right)
$$

where $\delta_{k k^{\prime}}$ is the Kronecker delta symbol and $\delta\left(m-m^{\prime}\right)$ the Dirac delta function.

We see that states of the quantum field with different frequencies, that is different masses, are orthogonal. Indeed as long as we introduce no $s$-dependence the one particle Hilbert space $\mathcal{H}_{1}$ and hence the N-particle Hilbert space $\mathcal{H}_{N}$ are decomposed into orthogonal superselection sectors labelled by the mass $m$ and particle number $N[3]$.

From the point of view of non-relativistic quantum mechanics in the lower dimensional Newton-Cartan spacetime we have an example of the much discussed fact [42] that the mass in non-relativistic quantum mechanics may be used to label super-selection sectors.

\section{SYMMETRIES OF DAMPED OSCILLATORS}

A simple example of a dissipative system is provided by the damped harmonic oscillator in one dimension, whose Hamiltonian [consistent with (II.6)] is

$$
H=\frac{1}{2 m} e^{-\gamma t} p^{2}+\frac{1}{2} e^{\gamma t} m \omega^{2} x^{2} .
$$

The aim of this section is to provide a geometrical description of its not-entirelystandard symmetry algebra using the Eisenhart (Bargmann) technique, combined with the approach of [8-11]. We begin by describing the Arnold transformation in these terms.

\section{A. The Arnold transformation}

The key concept followed in [8-11] is the Arnold Transformation [18], which takes a linear second-order differential equation into a free particle [18]. Its quantum extension was given as a unitary map between the space of solutions of the two systems and their operators [8].

The most general linear second-order differential equation in one dimension is,

$$
\ddot{x}+\dot{f}(t) \dot{x}+\omega^{2}(t) x=F(t),
$$

where $f, \omega$ and $F$ are functions of time, $t$. For the damped oscillator, $f(t)=\gamma t$ with $\gamma$ and $\omega$ constants and $F=0$. However the results of this section apply to a generic time dependence. 
Let $u_{1}, u_{2}$ be two independent solutions of the associated homogeneous equation and $u_{p}$ a particular solution of the full equation. It is convenient to choose the initial conditions as

$$
u_{1}\left(t_{0}\right)=\dot{u}_{2}\left(t_{0}\right)=u_{p}\left(t_{0}\right)=\dot{u}_{p}\left(t_{0}\right)=0, \quad \dot{u}_{1}\left(t_{0}\right)=u_{2}\left(t_{0}\right)=1
$$

Their Wronskian is $\dot{u}_{1} u_{2}-u_{1} \dot{u}_{2}=e^{-f}$. Then setting

$$
\xi=\frac{x-u_{p}}{u_{2}}, \quad \tau=\frac{u_{1}}{u_{2}},
$$

carries the motion into that of a free particle,

$$
\xi(\tau)=a \tau+b
$$

The transformation (III.91) is only local in general, as $t$ is allowed to vary between two consecutive zeros of $u_{2}(t)$. Geometrically, it is realised in terms of the Eisenhart lift as follows. Setting

$$
\mathrm{g}_{a b} d x^{a} d x^{b}=e^{f} d x^{2}+2 d t d s-2 e^{f}\left(\frac{1}{2} \omega^{2} x^{2}-F x\right) d t^{2},
$$

we recognize the metric (II.18) of the 3-dimensional Bargmann manifold $B^{3}$, with

$$
\alpha=e^{-f}, \quad \beta=\alpha^{-1}, \quad \frac{V}{m}=\frac{1}{2} \omega^{2} x^{2}-F(t) x .
$$

Then (III.91), completed with

$$
\sigma=s+e^{f} u_{2}\left(\frac{1}{2} \dot{u}_{2} \sigma^{2}+\dot{u}_{p} \sigma\right)+g(t) \quad \text { where } \quad \dot{g}(t)=\frac{1}{2} e^{t}\left(\dot{u}_{p}^{2}-\omega^{2} u_{p}^{2}+2 F u_{p}\right)
$$

allows us to rewrite (III.93) as ${ }^{5}$,

$$
\mathrm{g}_{a b} d x^{a} d x^{b}=e^{f} u_{2}^{2}\left(d \xi^{2}+2 d \tau d \sigma\right) .
$$

Thus the Arnold transformation makes manifest the conformal flatness of the Eisenhart metric for a one-dimensional forced simple harmonic oscillator with time-dependent friction term and frequency (III.89), generalizing earlier results [27, 28] to the frictional case.

Explicitly, the Arnold transformation for the damped harmonic oscillator is obtained by setting $e^{f}=e^{\gamma t}, F(t)=0$, choosing $u_{p} \equiv 0$ and

$$
u_{1}=e^{-\gamma t / 2} \frac{\sin \Omega t}{\Omega}, \quad u_{2}=e^{-\gamma t / 2}\left(\cos \Omega t+\frac{\gamma}{2 \Omega} \sin \Omega t\right), \quad \Omega^{2}=\omega^{2}-\gamma^{2} / 4,
$$

\footnotetext{
${ }^{5}$ A formula similar to (III.96) was conjectured by Aldaya et al [43].
} 
which satisfy the initial conditions (III.90). This provides us with

$$
\begin{aligned}
\xi & =\frac{e^{\gamma t / 2} x}{\cos \Omega t+\frac{\gamma}{2 \Omega} \sin \Omega t}, \\
\tau & =\frac{\sin \Omega t}{\Omega\left(\cos \Omega t+\frac{\gamma}{2 \Omega} \sin \Omega t\right)}, \\
\sigma & =s-\frac{1}{2} e^{\gamma t} x^{2} \frac{\omega^{2}}{\Omega} \frac{\sin \Omega t}{\cos \Omega t+\frac{\gamma}{2 \Omega} \sin \Omega t} .
\end{aligned}
$$

In the undamped case $\gamma=0$ (III.98) reduces to that of Niederer [44], lifted to Bargmann space [27, 28, 45, 46],

$$
\xi=\frac{x}{\cos \omega t}, \quad \tau=\frac{\tan \omega t}{\omega}, \quad \sigma=s-\frac{1}{2} x^{2} \omega \tan \omega t .
$$

Note that each half-period of the oscillator is mapped onto the full time axis, allowing one to recover the Maslov correction at the quantum level [27].

\section{Symmetries of the CK oscillator}

The geodesic Hamiltonian on the cotangent bundle of the Bargmann manifold $T^{*} B^{3}$ is the Eisenhart lift of (III.88),

$$
\mathcal{H}=\frac{1}{2} g^{a b} p_{a} p_{b}=\frac{1}{2} e^{-\gamma t} p_{x}^{2}+\frac{1}{2} e^{\gamma t} \omega^{2} x^{2} p_{s}^{2}+p_{t} p_{s},
$$

On the other hand, the free Hamiltonian in 1 space dimension lifted to its Bargmann space [which is simply Minkowski space in $1+2$ dimensions] is,

$$
\overline{\mathcal{H}}=\frac{1}{2} p_{\xi}^{2}+p_{\tau} p_{\sigma}
$$

Now our clue is that eq. (III.96) allows us to infer that the geodesic Hamiltonian $\mathcal{H}$ and $\overline{\mathcal{H}}$, the pull-back of the free Hamiltonian to $B^{3}$, are conformally related,

$$
\overline{\mathcal{H}}=e^{\gamma t} u_{2}^{2} \mathcal{H} .
$$

But conformally related metrics have identical conformal Killing vectors, which translates into a statement about conserved quantities for the two Hamiltonians: since classical motions lift to null geodesics in Bargmann space, conformally related Hamiltonians have identical symmetries.

Now the free particle is known to carry an extended Schrödinger symmetry [47-49] 6 . Imitating what is done in the friction-less case $[27,28,45,46]$, its generators can be "imported" 7 to the damped oscillator by means of the Arnold transformation (III.98).

\footnotetext{
${ }^{6}$ The "Schrödinger" symmetry has actually been found by Jacobi [50], see [51].

7 The words "import" and "export" signify pull-back and push-forward respectively by the Arnold map.
} 
Let us recall that the Bargmann group of the free particle is generated by the conserved quantities (components of the moment map) $T=p_{\xi}, E=-p_{\tau}, m=p_{\sigma}$ associated with $\xi \tau$ and $\sigma$-translations; boost are generated by $B=-p_{\sigma} \xi+p_{\xi} \tau$. Expressed in terms of the original variables, we have,

$$
\begin{aligned}
& p_{\xi}=u_{2} p_{x}-e^{\gamma t} \dot{u}_{2} x p_{s}, \\
& p_{\tau}=e^{\gamma t} u_{2} \dot{u}_{2} x p_{x}+u_{2}^{2} e^{\gamma t} p_{t}-\frac{1}{2} e^{2 \gamma t}\left(\dot{u}_{2}^{2}-\omega^{2} u_{2}^{2}\right) x^{2} p_{s}, \\
& p_{\sigma}=p_{s}=m,
\end{aligned}
$$

from which we infer, in particular,

$$
\overline{\mathcal{H}}=E=-p_{\tau}, \quad B=u_{1} p_{x}-e^{\gamma t} \dot{u}_{1} x p_{s} .
$$

The remaining Schrödinger generators, namely dilations and expansions are given by

$$
\begin{aligned}
D & =-2 \tau p_{\tau}-\xi p_{\xi} \\
& =-2 e^{\gamma t} u_{1} u_{2} p_{t}-\left(1+2 e^{\gamma t} u_{1} \dot{u}_{2}\right) x p_{x}+\frac{e^{\gamma t}}{u_{2}}\left[\dot{u}_{2}-e^{\gamma t} u_{1}\left(\omega^{2} u_{2}^{2}-\dot{u}_{2}^{2}\right)\right] x^{2} p_{s} \\
K & =\tau^{2} p_{\tau}+\tau \xi p_{\xi}-\frac{1}{2} \xi^{2} p_{s} \\
& =e^{\gamma t} u_{1}^{2} p_{t}+\frac{u_{1}}{u_{2}}\left(1+e^{\gamma t} u_{1} \dot{u}_{2}\right) x p_{x}+\frac{1}{2 u_{2}^{2}}\left[-1-2 e^{\gamma t} u_{1} \dot{u}_{2}+e^{2 \gamma t} u_{1}^{2}\left(\omega^{2} u_{2}^{2}-\dot{u}_{2}^{2}\right)\right] x^{2} p_{s} .
\end{aligned}
$$

The "exported" generators $T, B, E, m$ satisfy the (extended) Newton-Hooke Poisson algebra [25]; adding $D$ and $K$ one gets, once again, the Schrödinger algebra $[45,46]^{8}$.

Let us underline that some of the "imported" Schrödinger generators are explicitly $t$-dependent and are conserved only in the larger sense, i.e. for $f=T, B, m$ they satisfy $\{\mathcal{H}, f\}=0$, which in terms of 1-dimensional Poisson brackets expands as

$$
\{H, f\}_{1 d}+\frac{\partial f}{\partial t}=0 .
$$

The quantity $E$ instead is conserved only on-shell, satisfying

$$
\{\mathcal{H}, E\}=e^{\gamma t} u_{2}\left(\gamma u_{2}+2 u_{2}^{\prime}\right) \mathcal{H} .
$$

In conclusion, the damped oscillator shares (as does its friction-less cousin [44]), the Schrödinger symmetry of a free particle, despite the rather different form of the generators. It is important to stress, though, that the geodesic Hamiltonian $\mathcal{H}$ in (III.100) which generates the dynamics, does not belong to the symmetry algebra, whereas the "imported" free Hamiltonian $\overline{\mathcal{H}}$ in (III.101) does belong to the symmetry algebra but does not generate the dynamics.

\footnotetext{
${ }^{8}$ Upon the substitutions $p_{s} \rightarrow m, p_{x} \rightarrow-i \hbar \partial_{x}$ the quantities $T$ and $B$ are mapped to the operators $\hat{P}$ and $m \hat{X}$ of the quantum theory described in [8], explaining the origin of the Heisenberg-Weyl algebra found in [8]. $p_{\tau}$ is mapped to $-\frac{1}{2 m} \hat{P}^{2}$. Similarly, the quantities in (III.105) are linear combinations of the operators $\hat{X}^{2}, \hat{P}^{2}, \widehat{X P}$.
} 


\section{B. The Bateman "Doppelgänger" (Bateman double)}

The idea of Bateman [16] was to derive an action principle for the equation

$$
\ddot{x}+\gamma \dot{x}+\omega^{2} x=0
$$

by introducing an auxiliary field $y(t)$ as a Lagrange multiplier,

$$
S=\int y\left(\ddot{x}+\gamma \dot{x}+\omega^{2} x\right) d t .
$$

Variation w.r.t. $y$ reproduces (III.108), whereas variation w.r.t. $x$ yields a $y$-oscillator with "anti-damping",

$$
\ddot{y}-\gamma \dot{y}+\omega^{2} y=0 \text {. }
$$

Then

$$
S \sim \int L d t=m \int\left(\dot{x} \dot{y}-\frac{\gamma}{2}(y \dot{x}-x \dot{y})-\omega^{2} x y\right) d t
$$

where " $\sim$ " means up to surface terms. The associated momenta are defined as $p_{x}=\partial L / \partial \dot{x}$ and $p_{y}=\partial L / \partial \dot{y}$, leading to the Hamiltonian

$$
H_{B}=\frac{p_{x} p_{y}}{m}+\frac{\gamma}{2}\left(y p_{y}-x p_{x}\right)+m \Omega^{2} x y .
$$

Despite the appearances, the Hamiltonian $H_{B}$ gives rise to the equations of two noninteracting damped/anti-damped simple harmonic oscillators whose total energy $H_{B}$ is constant. Note that $H_{B}$ is indefinite.

Amusingly, introducing $u=\frac{x+y}{2} v=\frac{x-y}{2}$ eqns (III.108)-(III.110) can also be presented as two, coupled oscillators

$$
\begin{aligned}
& \ddot{u}+\omega^{2} u=-\gamma \dot{v} \\
& \ddot{v}+\omega^{2} v=-\gamma \dot{u}
\end{aligned}
$$

whose individual energies change at same rate,

$$
\begin{aligned}
& \frac{1}{m} \frac{d\left(E_{u}\right)}{d t}=\frac{d}{d t}\left(\frac{1}{2} \dot{u}^{2}+\omega^{2} u^{2}\right)=-\gamma \dot{u} \dot{v} \\
& \frac{1}{m} \frac{d\left(E_{v}\right)}{d t}=\frac{d}{d t}\left(\frac{1}{2} \dot{v}^{2}+\omega^{2} v^{2}\right)=-\gamma \dot{u} \dot{v}
\end{aligned}
$$

such that the total energy $H_{B}=E_{u}-E_{v}$ is indeed conserved.

One may wonder if the Bateman procedure can be extended to more general potentials $V$. The analog of eqn (III.87) would then be

$$
L=\dot{x} \dot{y}-\frac{\gamma}{2}(y \dot{x}-x \dot{y})-\frac{y}{m} V^{\prime}(x)
$$

which would yield the correct $x$-equation

$$
\ddot{x}+\gamma \dot{x}+\frac{V^{\prime}(x)}{m}=0
$$


cf. (III.108) but whose $y$-equation would be

$$
\ddot{y}-\gamma \dot{y}+\frac{y}{m} V^{\prime \prime}(x)=0 .
$$

The system is conservative but would consist of two be coupled equation, not symmetric in $x$ and $y$.

\section{Symmetries of the Bateman system}

The Bateman system of is composed of two uncoupled Caldirola-Kanai oscillators with equal masses and frequencies but opposites values of $\gamma$, with Hamiltonian

$$
H_{C K}=e^{-\gamma t} \frac{p_{x}^{\prime 2}}{2 m}+\frac{1}{2} m \omega^{2} x^{\prime 2} e^{\gamma t}-e^{\gamma t} \frac{p_{y}^{\prime 2}}{2 m}-\frac{1}{2} m \omega^{2} y^{\prime 2} e^{-\gamma t} .
$$

The transformation from the latter to the former is canonical and obtained by the following generating function of type-2 of mixed variables $F_{2}=F_{2}\left(x^{\prime}, y^{\prime}, p_{x}, p_{y}\right)$,

$$
p_{x}^{\prime}=\frac{\partial F_{2}}{\partial x^{\prime}}, \quad p_{y}^{\prime}=\frac{\partial F_{2}}{\partial y^{\prime}}, \quad \hat{x}=\frac{\partial F_{2}}{\partial \hat{p}_{x}}, \quad \hat{y}=\frac{\partial F_{2}}{\partial \hat{p}_{y}}, \quad H_{B}=H_{C K}+\frac{\partial F_{2}}{\partial t},
$$

where $H_{B}$ is (III.111) and

$$
F_{2}=\frac{1}{\sqrt{2}}\left(e^{\gamma t} x^{\prime}+y^{\prime}\right) p_{y}+\frac{1}{\sqrt{2}}\left(x^{\prime}-e^{-\gamma t} y^{\prime}\right) p_{x}+\frac{\gamma}{4 m \Omega^{2}} e^{-\gamma t} p_{x}^{2}-\frac{m \gamma}{8}\left(e^{\frac{\gamma t}{2}} x^{\prime}-e^{-\frac{\gamma t}{2}} y^{\prime}\right)^{2}
$$

Explicitly,

$$
\begin{aligned}
x & =\frac{1}{\sqrt{2}}\left[\frac{\omega^{2}}{\Omega^{2}}\left(x^{\prime}-e^{-\gamma t} y^{\prime}\right)+\frac{\gamma}{2 m \Omega^{2}}\left(e^{-\gamma t} p_{x}^{\prime}-p_{y}^{\prime}\right)\right], \\
y & =\frac{1}{\sqrt{2}}\left(e^{\gamma t} x^{\prime}+y^{\prime}\right), \\
p_{x} & =\frac{1}{\sqrt{2}}\left[p_{x}^{\prime}-e^{\gamma t} p_{y}^{\prime}+\frac{m \gamma}{2}\left(e^{\gamma t} x^{\prime}-y^{\prime}\right)\right], \\
p_{y} & =\frac{1}{\sqrt{2}}\left(p_{y}^{\prime}+e^{-\gamma t} p_{x}^{\prime}\right) .
\end{aligned}
$$

This transformation is defined in phase space and is not induced by a transformation acting on configuration space. In particular, there is no transformation between the Eisenhart lift of the Bateman system and that of the double Caldirola-Kanai

$$
\mathcal{H}=H_{C K}+p_{t} p_{s}
$$

In relation to (III.121) section III A provides us with two copies of the Bargmann (extended Galilei) algebra, one for $+\gamma$ and one for $-\gamma$. With the notations of III A we 
define $T_{1}=T, B_{1}=B$ and $E_{1}=E$ for the first copy. Then, we define the functions $v_{1,2}$ as the functions obtained from $u_{1,2}$ by changing $\gamma \rightarrow-\gamma$. In terms of these we define

$$
\begin{aligned}
& T_{2}=v_{2} p_{y}^{\prime}-e^{-\gamma t} \dot{v}_{2} y^{\prime} p_{s} \\
& B_{2}=v_{1} p_{y}^{\prime}-e^{-\gamma t} \dot{v}_{1} y^{\prime} p_{s} \\
& E_{2}=-e^{-\gamma t} v_{2} \dot{v}_{2} y^{\prime} p_{y}^{\prime}-v_{2}^{2} e^{-\gamma t} p_{t}+\frac{1}{2} e^{-2 \gamma t}\left(\dot{v}_{2}^{2}-\omega^{2} v_{2}^{2}\right) y^{\prime 2} p_{s} .
\end{aligned}
$$

The two copies of the Heisenberg subalgebra are mutually commuting:

$$
\begin{gathered}
\left\{T_{1}, T_{2}\right\}=0=\left\{T_{1}, B_{2}\right\}, \\
\left\{B_{1}, T_{2}\right\}=0=\left\{B_{1}, B_{2}\right\} .
\end{gathered}
$$

However, the two Bargmann algebras interact through the energy generators.

Acting repeatedly with $E_{1}$ on the $\left(T_{2}, B_{2}\right)$ Heisenberg subalgebra generates infinite copies of Heisenberg subalgebras due to the time-dependency of the generators, and similarly exchanging $1 \leftrightarrow 2$. By this we mean that if we define for $i \neq j, i, j=1,2$

$$
\begin{aligned}
\left\{E_{i}, T_{j}\right\}:=\tau_{j}^{(1)} & \left\{E_{i}, \tau_{j}^{(n)}\right\}:=\tau_{j}^{(n+1)} \\
\left\{E_{i}, B_{j}\right\}:=\beta_{j}^{(1)} & \left\{E_{i}, \beta_{j}^{(n)}\right\}:=\beta_{j}^{(n+1)}
\end{aligned}
$$

then we will have

$$
\left\{\tau_{i}^{(n)}, \beta_{j}^{(n)}\right\}=\delta_{i j} \mu_{i}^{(n)},
$$

for a time dependent $\mu_{i}^{(n)}$ proportional to $p_{s}$. These new Heisenberg subalgebras are also mutually commuting, as in (III.123).

For concreteness, specialising to the first $n=1$ level we find the following generators:

$$
\begin{aligned}
& \tau_{2}^{(1)}=e^{\gamma t} u_{2}^{2} \dot{v}_{2} p_{y}^{\prime}+\omega^{2} u_{2}^{2} v_{2} y^{\prime} p_{s}, \\
& \tau_{1}^{(1)}=e^{-\gamma t} v_{2}^{2} \dot{u}_{2} p_{x}^{\prime}+\omega^{2} v_{2}^{2} u_{2} x^{\prime} p_{s}, \\
& \beta_{2}^{(1)}=e^{\gamma t} u_{2}^{2} \dot{v}_{1} p_{y}^{\prime}+\omega^{2} u_{2}^{2} v_{1} y^{\prime} p_{s}, \\
& \beta_{1}^{(2)}=e^{-\gamma t} v_{2}^{2} \dot{u}_{1} p_{x}^{\prime}+\omega^{2} v_{2}^{2} u_{1} x^{\prime} p_{s}, \\
& \mu_{2}^{(1)}=e^{2 \gamma t} \omega^{2} u_{2}^{4} p_{s}, \\
& \mu_{1}^{(2)}=e^{-2 \gamma t} \omega^{2} v_{2}^{4} p_{s} .
\end{aligned}
$$

In other words if we decide to measure time using the coordinate $\tau_{1}=\frac{u_{1}}{u_{2}}$ associated to the generator $E_{1}$, then our physical system will be described by a standard Heisenberg algebra $\left(T_{1}, B_{1}\right)$ for a 'freely falling' first particle, plus another time dependent Heisenberg algebra $\left(T_{2}, B_{2}\right)$ whose generators have a non-standard dependence on time that gives rise to the infinite set of copies of Heisenberg algebras. The remaining brackets are between 
elements of the $\{T, B\}$ and $\{\tau, \beta\}$ algebras:

$$
\begin{gathered}
\left\{T_{1}, \tau_{2}^{(1)}\right\}=0, \quad\left\{T_{1}, \tau_{1}^{(1)}\right\}=\alpha_{1}, \quad\left\{B_{1}, \tau_{2}^{(1)}\right\}=0, \quad\left\{B_{1}, \tau_{1}^{(1)}\right\}=\beta_{1}, \\
\left\{T_{1}, \beta_{2}^{(1)}\right\}=0, \quad\left\{T_{1}, \beta_{1}^{(1)}\right\}=\beta_{1}, \quad\left\{B_{1}, \beta_{2}^{(1)}\right\}=0, \quad\left\{B_{1}, \beta_{1}^{(1)}\right\}=\gamma_{1}, \\
\left\{T_{2}, \tau_{2}^{(1)}\right\}=\alpha_{2}, \quad\left\{T_{2}, \tau_{1}^{(1)}\right\}=0, \quad\left\{B_{2}, \tau_{2}^{(1)}\right\}=\beta_{2}, \quad\left\{B_{2}, \tau_{1}^{(1)}\right\}=0 \\
\left\{T_{2}, \beta_{2}^{(1)}\right\}=\beta_{2}, \quad\left\{T_{2}, \beta_{1}^{(1)}\right\}=0, \quad\left\{B_{2}, \beta_{2}^{(1)}\right\}=\gamma_{2}, \quad\left\{B_{2}, \beta_{1}^{(1)}\right\}=0
\end{gathered}
$$

where

$$
\begin{aligned}
& \alpha_{1}=-v_{2}^{2}\left(\omega^{2} u_{2}^{2}+\dot{u}_{2}^{2}\right) p_{s}, \\
& \beta_{1}=-v_{2}^{2}\left(\omega^{2} u_{1} u_{2}+\dot{u}_{1} \dot{u}_{2}\right) p_{s}, \\
& \gamma_{1}=-v_{2}^{2}\left(\omega^{2} u_{1}^{2}+\dot{u}_{1}^{2}\right) p_{s}
\end{aligned}
$$

and where $\alpha_{2}, \beta_{2}, \gamma_{2}$ are obtained by the interchange $u \leftrightarrow v$. These elements commute with all the elements in the algebra with the exclusion of $E_{1}$ and $E_{2}$. Acting with the latter gives rise to an infinite tower of elements proportional to $p_{s}$.

Lastly: if we now consider dilations $D_{1,2}$ and expansions $K_{1,2}$ in the algebra we get again new terms. However, all of these are of similar nature to the ones found in this section: because of the $S L(2, \mathbb{R})$ symmetry we get algebras similar to the ones above if instead of $E_{1,2}$ we use $D_{1,2}$ or $K_{1,2}$. Algebraically this happens as different permutations of the $u_{1,2}, v_{1,2}$ functions are allowed.

\section{HUBBLE FRICTION}

Our ultimate example of a universal non-autonomous influence on all physical systems is the expansion of the universe. While the background metric may have a high degree of spatial symmetry, it certainly lacks time translation invariance except in the special cases of Minkowski spacetime and Einstein-Static universe. In the case of the de-Sitter and anti-de-Sitter spacetimes the spacetime is in fact of maximal symmetry, like Minkowski spacetime, but this fact is disguised by the fact that they may be cast in an expanding Friedmann-Lemaître form:

$$
g_{\mu \nu} d x^{\mu} d x^{\nu}=-c^{2} d t^{2}+a^{2}(t) g_{i j}\left(q^{k}\right) d q^{i} d q^{j}
$$

where $x^{\mu}\left(t, q^{k}\right)$ and $g_{i j}\left(q^{k}\right)$ is the maximally symmetric metric on $\mathbb{E}^{3}, S^{3}$ or $H^{3}$. The obvious notion of energy is not conserved in such spacetimes and if expanding this leads to a universal source of dissipation, an example of which is the phenomenon of Hubble friction which we shall now examine using the ideas developed in this paper. 


\section{A. Particle moving in an inhomogeneous cosmological spacetime}

We shall begin by considering the motion of a free particle moving in a spatially flat Friedmann-Lemaitre spacetime with scale factor $a=a(t)$ and metric

$$
g_{\mu \nu} d x^{\mu} d x^{\nu}=-c^{2} d t^{2}+a^{2}(t) d \boldsymbol{r}^{2},
$$

where $t$ is cosmic time. The Lagrangian and equation of motion for a relativistic point particle moving in the space-time (IV.130) are

$$
L=-m \int d t \sqrt{1-\frac{a^{2} \dot{\boldsymbol{r}}^{2}}{c^{2}}} \text { and } \frac{d}{d t}\left(a^{2} \frac{\dot{\boldsymbol{r}}}{\sqrt{1-\frac{a^{2} \dot{r}^{2}}{c^{2}}}}\right)=0,
$$

respectively. The spatial coordinate $\boldsymbol{r}$ here is called co-moving position since the world lines of the matter (e.g. galaxies participating in the Hubble flow) have $\boldsymbol{r}=$ constant. In the Newtonian limit the quantity $\boldsymbol{x}=a(t) \boldsymbol{r}$ corresponds to an inertial coordinate. For an account of Newtonian cosmology from a point particle perspective and a review of earlier fluid-based models the reader may consult $[32,52-54]$.

To obtain the Newtonian limit we let $c \rightarrow \infty$ and find the equation of motion

$$
\frac{d\left(a^{2} \dot{\boldsymbol{r}}\right)}{d t}=0 \quad \Rightarrow \quad \ddot{\boldsymbol{r}}+2 \frac{\dot{a}}{a} \dot{\boldsymbol{r}}=0 .
$$

Clearly, if $\dot{a}>0$ the "free" particle experiences a universal (i.e. mass-independent) frictional force. In general the friction coefficient $\gamma=2 \dot{a} / a$ is time dependent. However if $a(t)=e^{H t}$, where $H=$ const., which corresponds to spatially flat de-Sitter universe, $\gamma=2 H$, and the coefficient is independent of cosmic time $t$.

In the general case we may always introduce

$$
\tau=\int \frac{d t}{a^{2}},
$$

for which the friction coefficient vanishes. This example reinforces the idea that the notion of dissipation depends on the time coordinate one uses.

The Friedmann-Lemaitre metric with additional matter distribution is given approximately by the McVittie metric $[55,56]$ cf. $[57,58]$

$$
d s^{2}=-\left(1+\frac{2 U(x)}{a(t)}\right) d t^{2}+a^{2}(t)\left(1-\frac{2 U(x)}{a(t)}\right) g_{i j} d x^{i} d x^{j},
$$

where $U(x)$ is the Newtonian potential satisfying $\nabla_{g}^{2} U=4 \pi \delta \rho . \nabla_{g}^{2}$ is the Laplace-Beltrami operator with respect to the spatial metric $g_{i j}$, which is of constant curvature $k$, and the scale factor $a(t)$ satisfies the Friedmann equation

$$
\frac{\dot{a}^{2}}{a^{2}}+\frac{k}{a^{2}}=\frac{8 \pi}{3} \rho(t)
$$

with $\rho(t)$ the background energy density.

For point particles of mass $m_{a} U\left(x^{i}\right)=-\sum_{b} G\left(x^{i}, x_{b}^{j}\right)$ where $G\left(x^{i}, x_{b}^{j}\right)$ is the Green's function $-\nabla_{g}^{2} U=4 \pi \sum_{b} \delta\left(x, x_{b}\right)$. For a single particle and certain energy densities, e.g. $\rho$ constant and $k=0$, the McVittie metric is exact. 


\section{B. The Dmitriev-Zel'dovich equations}

If we consider more than one particle, we can take into account their gravitational interactions. The motion is then governed by the Dmitriev-Zel'dovich equations [59, 60] considered earlier in [5] in the present context. For a derivation from Newtonian gravity and and references to earlier work see $[53,61])$.

In our notation ${ }^{9}$ the Dmitriev-Zel'dovich equations read

$$
\ddot{\boldsymbol{r}}_{a}+2 \frac{\dot{a}}{a} \dot{\boldsymbol{r}}_{a}=\sum_{b \neq a} \frac{G m_{b}\left(\boldsymbol{r}_{b}-\boldsymbol{r}_{a}\right)}{a(t)^{3}\left|\boldsymbol{r}_{a}-\boldsymbol{r}_{b}\right|^{3}},
$$

where the Lagrangian from which the Dmitriev-Zel'dovich equations may be obtained, namely equation (63) of [53], is

$$
L=\sum_{a} \frac{1}{2} a^{2}(t) m_{a} \dot{\boldsymbol{r}}_{a}^{2}-\frac{1}{a(t)} V \quad \text { where } \quad V=-\sum_{1 \leq a \leq b \leq N} \frac{G m_{a} m_{b}}{\left|\boldsymbol{r}_{a}-\boldsymbol{r}_{b}\right|} .
$$

This eqn is of the Caldirola-Kanai form (II.6), with

$$
\alpha(t)=\frac{1}{a^{2}(t)}, \quad \beta(t)=\frac{1}{a(t)} .
$$

The quickest way of obtaining the Lagrangian (IV.137) is to consider a time-like geodesic of the McVittie metric (IV.134) in the non-relativistic limit. In General Relativity, Newton's constant $G$ is taken to be independent of both time and space. However in the context of this paper it is natural to suppose that it might vary with time as was suggested by Dirac [62]. Indeed, this was one of the original motivations for [5]. Following the

observation of [63] that limits on $\dot{G} / G$ may be obtained using the binary pulsar it become desirable to understand better the results of [64-66] on the symmetries of the equations of motion of gravitating bodies when $G$ is time dependent. The best current upper limits $\left(-1.7 \times 10^{-12} \mathrm{yr}^{-1}<\dot{G} / G<0.5 \times 10^{-12} \mathrm{yr}^{-1}\right.$ at $95 \%$ confidence level $)$ come from 21 years of observations of the binary pulsar [67]. In what follows we shall allow $G$ to have arbitrary time dependence.

Turning to symmetries, we note that, in addition to the obvious translation and rotation invariance, equations (IV.136) admit an analogue of the Galilei invariance of a similar type to the one which is responsible for Kohn's theorem [25, 46]. That is, given a solution $\boldsymbol{r}(t)$ of (IV.136), then

$$
\tilde{\boldsymbol{r}}_{a}(t)=\boldsymbol{r}_{a}(t)+\boldsymbol{r}(t)
$$

for $a=1, \ldots, N$ is also a solution so long as $\boldsymbol{r}(t)$ is a solution of (IV.132), - which is in fact (IV.136) with the interaction terms switched off. The general solution of (IV.132) is

$$
\boldsymbol{r}(t)=\mathbf{a}+\boldsymbol{u} \tau, \quad \tau=\int_{0}^{t} \frac{d t^{\prime}}{a^{2}\left(t^{\prime}\right)},
$$

${ }^{9} S(t)$ is our $a(t)$. 
where $\mathbf{a}$ and $\boldsymbol{u}$ are constant vectors. Thus if $\boldsymbol{u}=0$ we recover translation invariance and if $\mathbf{a}=0$, we obtain a generalization of Galilean boosts, which reduce precisely to the latter if the scale factor $a(t)$ is constant. Therefore, because time translation invariance is broken in the general case, we obtain a 9-parameter group of symmetries rather than the full Galilei group with all of its 10 parameters.

Can this group of symmetries be further extended ? We note that

$$
\mathbf{P}=\frac{\partial}{\partial \boldsymbol{r}}, \quad \mathbf{K}=\tau \frac{\partial}{\partial \boldsymbol{r}}
$$

do indeed generate the Abelian group (IV.140). However, while $\mathbf{P}$ commutes with the Hamiltonian, $[H, \mathbf{P}]=0$,

$$
[H, \mathbf{K}]=\frac{1}{a^{2}} \mathbf{P}=\mathbf{K}_{1}
$$

is a new, generally time-dependent generator. It is clear that taking further commutators with $H$ will lead to the introduction of an infinite number of generalized boosts, $\mathbf{K}_{l}, l=$ $1,2, \ldots$, and hence an infinite dimensional deformation of the Galilean algebra.

\section{Bianchi cosmology}

One may easily generalise the previous discussion to the case of homogeneous cosmologies of Bianchi type.

For these cosmologies $g_{i j}\left(q^{k}, t\right)$ is a time-dependent left-invariant metric on of the nine 3-dimensional Lie groups first classified by Bianchi. Using the notation of Sec. II D, the spacetime metric is of the form

$$
g_{\mu \nu} d x^{\mu} d x^{\nu}=-d t^{2}+g_{i j}\left(q^{k}, t\right) d q^{i} d q^{j}, \quad g_{i j}=B_{a b} \lambda_{i}^{a} \lambda_{j}^{b} .
$$

In the non-relativistic limit we have

$$
L=\frac{1}{2} g_{i j} \dot{q}^{i} \dot{q}^{j}
$$

The quantities

$$
N_{c}=B_{a b}(t) \lambda_{j}^{a} R_{c}^{j} \lambda_{i}^{b} \dot{q}^{i}
$$

are conserved. One has

$$
\dot{q}^{i}=L_{a}^{i} B^{a b}(t) L_{b}^{l} \rho_{j}^{c} N_{c}=g^{i j}\left(q^{k}, t\right) \rho_{j}^{c} N_{c},
$$

which shows that $B_{a b}(t)$ is responsible for anisotropic Hubble friction. The simplest example is that of Bianchi I for which $R_{i}^{a}=\delta_{i}^{a}=L_{i}^{a}$ and thus

$$
d s^{2}=-d t^{2}+g_{i j}(t) d q^{i} d q^{j}
$$

One finds that

$$
\dot{q}^{i}=g^{i j}(t) N_{j} \quad \Rightarrow \quad \ddot{q}^{i}=-\gamma_{j}^{i}(t) \dot{q}^{j},
$$

where $\gamma_{j}^{i}(t)=\dot{g}^{i k} g_{k j}$ is a time-dependent friction tensor.

Further details on Newtonian cosmology are presented in a companion paper [32]. 


\section{Acknowledgments}

MC thanks the Physics Department of the University of Camerino for hospitality, and the Brazilian funding agency CNPQ for funding under project 205029/2014-0. GWG is grateful to the Laboratoire de Mathématiques et de Physique Théorique de l'Université de Tours for hospitality and the Région Centre for a "Le Studium" research professorship. PAH acknowledges partial support from the Chinese Academy of Sciences' Presidential International Fellowship (Grant No. 2010T1J06) and the Institute of Modern Physics of the CAS at Lanzhou as well as the Instituto de Astrofísica de Andalucía for hospitality. $\mathrm{MC}$ and PAH also benefited from discussions with V. Aldaya, F. Cossío, J. Guerrero and F.F. López-Ruiz.

[1] L. P. Eisenhart, "Dynamical trajectories and geodesics", Annals. Math. 30 591-606 (1928).

[2] C. Duval, G. Burdet, H. P. Künzle and M. Perrin, "Bargmann Structures and NewtonCartan Theory," Phys. Rev. D 31 (1985) 1841.

[3] J. Gomis and J. M. Pons, "Poincare Transformations and Galilei Transformations," Phys. Lett. A 66 (1978) 463.

[4] A. P. Balachandran, H. Gomm and R. D. Sorkin, "Quantum Symmetries From Quantum Phases: Fermions From Bosons, a $Z(2)$ Anomaly and Galilean Invariance," Nucl. Phys. B $281(1987) 573$.

[5] C. Duval, G. W. Gibbons and P. Horvathy, "Celestial mechanics, conformal structures and gravitational waves," Phys. Rev. D 43 (1991) 3907 [hep-th/0512188].

[6] M. Cariglia, "Hidden Symmetries of Dynamics in Classical and Quantum Physics," Rev. Mod. Phys. 86 (2014) 1283 [arXiv:1411.1262 [math-ph]].

[7] D. C. Brody, G. W. Gibbons and D. M. Meier, "Time-optimal navigation through quantum wind," New J. Phys. 17 (2015) 033048 [arXiv:1410.6724 [quant-ph]]; “A Riemannian approach to Randers geodesics," arXiv:1507.08185 [math-ph].

[8] V. Aldaya, F. Cossío, J. Guerrero and F.F. López-Ruiz, "The quantum Arnold transformation," J. Phys. A, 44, 065302 (2011). arXiv:1010.5521

[9] V. Aldaya, F. Cossío, J. Guerrero, F.F. López-Ruiz "A symmetry trip from Caldirola to Bateman damped systems," arXiv:1102.0990 [math-ph]

[10] J. Guerrero, F. F. López-Ruiz, V. Aldaya, F. Cossío, "Symmetries of the quantum damped harmonic oscillator," J. Phys. A. arXiv:1210.4058 [math-ph] 
[11] J. Guerrero, V. Aldaya, F. F. López-Ruiz and F. Cossío, "Unfolding the quantum Arnold transformation," Int. J. Geom. Meth. Mod. Phys. 09 (2012) 02, 1260011.

[12] H. Dekker, "Classical and quantum mechanics of the damped harmonic oscillator," Phys. Rept. 80, 1-112 (1981)

[13] C. I. Um, K. H. Yeon and T. F. George, "The Quantum damped harmonic oscillator," Phys. Rept. 362 (2002) 63.

[14] P. Caldirola, "Forze non-conservative nella meccanica quantistica," Nuovo Cimento, 18, 393 (1941).

[15] E. Kanai, "On the Quantization of the Dissipative Systems" Prog. Theor. Phys., 3, 440 (1948).

[16] H. Bateman, "On dissipative systems and related variational principles," Phys. Rev. 38, $815(1931)$

[17] G. W. Gibbons, "Quantized Fields Propagating in Plane Wave Space-Times," Commun. Math. Phys. 45 (1975) 191.

[18] V. I. Arnold, Supplementary Chapters to the Theory of Ordinary Differential Equations (Nauka, Moscow, 1978); Geometrical Methods in the Theory of Ordinary Differential Equations (Springer-Verlag, New York, Berlin, 1983), in English.

[19] D. Cangemi and R. Jackiw, "Gauge Invariant Formulations Of Lineal Gravity," Phys. Rev. Lett. 69 (1992) 233 [arXiv:hep-th/9203056].

[20] C. R. Nappi and E. Witten, "WZW model based on a nonsemisimple group," Phys. Rev. Lett. 71, 3751 (1993), hep-th/9310112

[21] H. Goldstein, Classical Mechanics (2nd ed.) (1980) . Reading, MA: Addison-Wesley. p. 24.

[22] E. Minguzzi, "Rayleigh's dissipation function at work," Eur. J. Phys. 36 (2015) 035014 arXiv:1409.4041

[23] B. Hoffmann, "Kron's Non-Riemannian Electrodynamics," Rev. Mod. Phys. 21 (1949) $535-540$

[24] H. W. Brinkmann, "Einstein spaces which are mapped conformally on each other," Math. Ann. 94, 119 (1925).

[25] G. W. Gibbons and C. N. Pope, "Kohn's Theorem, Larmor's Equivalence Principle and the Newton-Hooke Group," Annals Phys. 326 (2011) 1760 [arXiv:1010.2455 [hep-th]].

[26] C. Duval and S. Lazzarini, "On the Schrödinger-Newton equation and its symmetries: a 
geometric view," Class. Quant. Grav. 32 (2015) 175006 [arXiv:1504.05042 [math-ph]].

[27] G. Burdet, C. Duval and M. Perrin, "Time-Dependent Quantum Systems and Chronoprojective Geometry", Lett. Math. Phys. 10 (1985) 255.

[28] C. Duval, P. A. Horváthy and L. Palla, "Conformal properties of Chern-Simons vortices in external fields," Phys. Rev. D50, 6658 (1994) [hep-ph/9405229, hep-th/9404047].

[29] A. Bravetti, H. Cruz, D. Tapias, "Contact Hamiltonian Mechanics," [ArXiv: 1604.08266 [math-ph]]

[30] J.-M. Souriau, Structure des systèmes dynamiques, Dunod (1970); Structure of Dynamical Systems. A Symplectic View of Physics, Birkhäuser, (1997).

[31] A. V. Aminova and N. A-M Aminov, "Projective geometry of systems of second-order differential equations," Sbornik Mathematics 197951 (2006).

[32] C. Duval, G. Gibbons and P. Horvathy, "Conformal and projective symmetries in Newtonian cosmology," [arXiv:1605.00231 [gr-qc]].

[33] J. Cerverò and J. Villarroel, "SL(3, R) realisations and the damped harmonic oscillator", J. Phys. A 17 (1984) 1777.

[34] A. Liénard, "Etude des oscillations entretenues," Revue générale de l'électricité, 23 901-912, 1928; ibid. 23 946-954, 1928.

[35] B. van der Pol, "On relaxation-oscillations," The London, Edinburgh and Dublin Philosophical Magazine and Journal of Science 2 978-992, (1927); B. van der Pol and J. van der Mark, "The heartbeat considered as a relaxation oscillation, and an electrical model of the heart," ibid. 6, 763-775, (1928).

[36] W. R. Hamilton, "The hodograph, or a new method of expressing in symbolic language the Newtonian law of attraction," Proc. Roy. Irish Acad. 3 (1847) 344.

[37] G. W. Gibbons and S. Gielen, "The Petrov and Kaigorodov-Ozsvath Solutions: Spacetime as a Group Manifold," Class. Quant. Grav. 25 (2008) 165009 [arXiv:0802.4082 [gr-qc]].

[38] G. W. Gibbons and C. M. Warnick, "The helical phase of chiral nematic liquid crystals as the Bianchi VII(0) group manifold, “ Phys. Rev. E 84 (2011) 031709 [arXiv:1106.2423 [gr-qc]].

[39] C. Duval, Z. Horvath and P. A. Horvathy, "The Nappi-Witten example and gravitational waves," [hep-th/9404018].

[40] H. Kyono and K. Yoshida, "Yang-Baxter invariance of the Nappi-Witten model," Nucl. 
Phys. B 905 (2016) 242 [arXiv:1511.00404 [hep-th]].

[41] V.I. Arnold, "Sur la géométrie différentielle des groupes de Lie de dimension infinie et ses applications à l'hydrodynamique des fluides parfaits," Ann. Inst. Fourier 16 (1966) 316.

[42] D. Giulini, "On Galilei invariance in quantum mechanics and the Bargmann superselection rule," Annals Phys. 249 (1996) 222 [quant-ph/9508002].

[43] V. Aldaya, F. Cossío, J. Guerrero and F. F. López-Ruiz, Private communication (2013).

[44] U. Niederer, "The maximal kinematical invariance group of the harmonic oscillator," Helv. Phys. Acta 46 (1973) 191.

[45] M. Hassaïne and P. A. Horvathy, "The Symmetries of the Manton superconductivity model," J. Geom. Phys. 34 (2000) 242 [math-ph/9909025].

[46] P. M. Zhang and P. A. Horvathy, "Kohn's theorem and Galilean symmetry," Phys. Lett. B 702 (2011) 177 [arXiv:1105.4401 [hep-th]].

[47] R. Jackiw, "Introducing scale symmetry," Phys. Today 25 (1972) 23.

[48] U. Niederer, "The maximal kinematical invariance group of the free Schrödinger equation." Helv. Phys. Acta 45, 802 (1972).

[49] C. R. Hagen, C. R. Hagen, "Scale and conformal transformations in galilean-covariant field theory," Phys. Rev. D 5 (1972) 377.

[50] C. G. J. Jacobi, "Vorlesungen über Dynamik." Univ. Königsberg 1842-43. Herausg. A. Clebsch. Vierte Vorlesung: Das Princip der Erhaltung der lebendigen Kraft. Zweite ausg. C. G. J. Jacobi's Gesammelte Werke. Supplementband. Herausg. E. Lottner. Berlin Reimer (1884).

[51] C. Duval and P. A. Horvathy, "Non-relativistic conformal symmetries and Newton-Cartan structures," J. Phys. A42, 465206 (2009).

[52] G. F. R. Ellis and G. W. Gibbons, "Discrete Newtonian Cosmology," Class. Quant. Grav. 31 (2014) 025003 [arXiv:1308.1852 [astro-ph.CO]].

[53] G. F. R. Ellis and G. W. Gibbons, "Discrete Newtonian Cosmology: Perturbations," Class. Quant. Grav. 32 (2015) 5, 055001 [arXiv:1409.0395 [gr-qc]].

[54] S. Benenti, "Mathematical models in isotropic cosmology", Working Paper April 2016. DOI: 10.13140/RG.2.1.191.8800

[55] G. C. McVittie, The mass-particle in an expanding universe," Mon. Not. Roy. Astron. Soc. 93 (1933) 325. 
[56] G C McVittie, General Relativity and Cosmology, $2^{\text {nd }}$ ed. Chapman and Hall (1964).

[57] R M Wald, "Gravitational Lensing in Inhomogeneous Universes," [gr-qc/9806097]

[58] D. E. Holz and R. M. Wald, "A New method for determining cumulative gravitational lensing effects in inhomogeneous universes," Phys. Rev. D 58 (1998) 063501 [astro-ph/9708036].

[59] N. A. Dmitriev and Ya. B. Zel'dovich, "The energy of accidental motions in the expanding universe," Sov. Phys. JETP 18793 (1964)

[60] P J E Peebles, "The Large-scale structure of the universe," Princeton University Press, Princeton, NJ (1980).

[61] G.W. Gibbons and C.E.Patricot, "Newton-Hooke spacetimes, Hpp-waves and the cosmological constant", Class. Quant. Grav. 20 (2003) 5225.

[62] P. A. M. Dirac, "The cosmological constants," Nature, 139 (1937) 323 "A new basis for cosmology," Proc. R. Soc. A165, 199 (1938)

[63] T. Damour, G. W. Gibbons and J. H. Taylor, "Limits on the Variability of G Using BinaryPulsar Data,” Phys. Rev. Lett. 61 (1988) 1151. doi:10.1103/Phys. Rev. Lett..61.1151

[64] J. Mestschersky, "Ein Spezialfall der Gyldén'schen Problem (A.N. 2593)," Astronomische Nachrichten 132 (3153) (1892) 129; "Ueber die Integration der Bewegungsgleichungen im Probleme zweier Körper von veränderlicher Masse", Astronomische Nachrichten 159 (3807) (1902) 231

[65] J.P. Vinti, "Classical solution of the two-body problem if the gravitational constant diminishes inversely with the age of the universe," Not. Roy. Astron. Soc. 169 (1974) 417

[66] D. Lynden-Bell, "On the $N$-body problem in Dirac's cosmology," Observatory 102, 86 (1982)

[67] W. W. Zhu, et al, "Testing Theories of Gravitation Using 21-Year Timing of Pulsar Binary J1713+0747," [arXiv:1504.00662 [astro-ph]]. 